Diesem Heft liegt das Bestellformular für die Einbanddecken 1983 zu dieser Zeitschrift bei.

(c) Verlag Chemie GmbH, D-6940 Weinheim 1984 - Printed in the Federal Republic of Germany.

Verantwortlich für den Inhalt: Prof. Dr. Hans Musso, Karlsruhe. Redaktion: Dr. Hermann Zahn, München.

Verlag Chemie GmbH (Geschäftsführer: Prof. Dr. Helmut Grünewald und Hans Dirk Köhler), Pappelallec 3, Postfach 1260/1280, D-6940 Weinheim.

Anzeigenleitung: R. J. Roth, Weinheim.

Die Wiedergabe von Gebrauchsnamen, Handelsnamen, Warenbezeichnungen und dgl. in dieser Zeitschrift berechtigt nicht zu der Annahme, daß solche Namen ohne weiteres von jedermann benutzt werden dürfen. Vielmehr handelt es sich häufig un gesetzlich geschützte eingetragene Warenzeichen, auch wenn sie nicht als solche gekennzeichnet sind.

Alle Rechte, insbesondere die der Übersetzung in fremde Sprachen, vorbehalten. Kein Teil dieser Zeitschrift darf ohne schriftliche Genehmigung des Verlages in irgendeiner Form - durch Photokopie, Mikrofilm oder irgendein anderes Verfahren -. reproduziert oder in eine von Maschinen, insbesondere von Datenverarbeitungsmaschinen verwendbare Sprache übertragen oder übersetzt werden. - All rights reserved (including those of translation into foreign languages). No part of this issue may be reproduced in any form - by photoprint, microfilm, or any other means - nor transmitted or translated into a machine language without the permission in writing of the publishers. - Von einzelnen Beiträgen oder Teilen von ihnen dürfen nur einzelne Vervielfäligungsstücke für den persönlichen oder sonstigen eigenen Gebrauch hergestellt werden. Jede im Bereich eines gewerblichen Unternehmens hergestellte oder benutzte Kopie dient gewerblichen Zwecken gem. \$ 54(2) UrhG und verpflichtet zur Gebührenzahlung an die VG WORT, Abteilung Wissenschaft, Goethestr. 49, 8000 München 2, von der die einzelnen Zahlungsmodalitäten zu erfragen sind. Die Weitergabe von Vervielfältigungen, gleichgültig zu welchem Zweck sie hergestellt werden, ist eine Urheberrechtsverletzung.

Valid for users in the U.S.A.: The appearance of the code at the bottom of the first page of an article in this journal (serial) indicates the copyright owner's consent that copies of the article may be made for personal or internal use, or for the personal or internal use of specific clients. This consent is given on the condition, however, that the copier pay the stated percopy fee through the Copyright Clearance Center, Inc., for copying beyond that permitted by Sections 107 or 108 of the U.S. Copyright Law. This consent does not extend to other kinds of copying, such as copying for general distribution, for advertising or promotional purposes, for creating new collective work, or for resale. For copying from back volumes of this journal see "Permissions to Photo-Copy: Publisher's Fee List " of the CCC.

In der Zeitschrift werden keine Rezensionen veröffentlicht; zur Besprechung eingehende Bücher werden nicht zurückgesandt. Herstellung: Krebs-Gehlen Druckerei, Hemsbach/Bergstraße. 


\section{CHEMISCHE BERICHTE}

GEGRÜNDET 1868

117. JAHRGANG

HERAUSGEGEBEN IM AUFTRAG DER GESELLSCHAFT DEUTSCHER CHEMIKER

VON

K. HAFNER - W. KIRMSE - H. MUSSO - H. NÖTH •

J. SAUER - E. WINTERFELDT

UNTER MITWIRKUNG VON

H. A. BRUNE · W. LÜTTKE - G. SPITELLER

REDAKTION: H. ZAHN

mit H. SCHILL, J. STREHLOW und A. WIELAND 


\section{BEMERKUNGEN DER REDAKTION}

1. Die Chemischen Berichte (zu zitieren als Chem. Ber.) setzen die Berichte der Deutschen Chemischen Gesellschaft (zu zitieren als Ber. Dtsch. Chem. Ges.) fort.

2. Die „Berichte“ enthalten Originalmitteilungen aus allen Gebieten der Chemie.

3. Die „Berichte“ erscheinen monatlich; ein Registerheft beschließt jeden Jahrgang.

4. Die Verantwortung für ihre Mitteilungen tragen die Verfasser selbst. - Der Korrespondenzautor ist durch einen hochgestellten Stern hervorgehoben. Die Zugehörigkeit der Autoren zu den Instituten ist durch hochgestellte Kleinbuchstaben markiert.

5. Es werden grundsätzlich nur Arbeiten aufgenommen, die vorher weder im Inland noch im Ausland veröffentlicht worden sind.

6. Eine Anweisung zur Abfassung von Manuskripten für die „Berichte“ wird auf Wunsch zugestellt.

7. Manuskripte sind zu senden an Redaktion der Chemischen Berichte. Dr. H. Zahn, Am Klopferspitz, D-8033 Martinsried, Telefon (089) 85783830.

8. Der Eingang der Abhandlungen wird den Autoren am Tage der Registrierung angezeigt.

9. Es werden nur Manuskripte in deutscher oder englischer Sprache aufgenommen. Allen Beiträgen ist eine knappe Zusammenfassung (summary) in beiden Sprachen voranzustellen, einschließlich Titel in der jeweils zweiten Sprache.

10. Der Autor muß das alleinige Urheberrecht besitzen. Mit der Annahme des Manuskriptes durch die Redaktion überträgt er dem Verlag Chemie das ausschließliche Nutzungsrecht, insbesondere das Recht der Vervielfältigung wie Fotokopie, Mikrofilm - oder mit irgendeinem anderen Verfahren - oder das Manuskript in eine von Maschinen, insbesondere von Datenverarbeitungsmaschinen, verwendbare Sprache zu übergeben oder zu übersetzen (auch in fremde Sprachen).

11. Den Autoren werden 75 Sonderdrucke unentgeltlich portofrei zugesandt. Wünscht ein Autor mehr als 75 Abzüge, so ist dies auf dem Manuskript oder spätestens bei Rücksendung der Korrektur auf dieser zu vermerken. Den Autoren werden nur die Selbstkosten für die Zahl der die Freiexemplare überschreitenden Sonderabzüge berechnet.

12. Anfragen nach dem Verbleib nicht eingetroffener Berichte-Hefte oder Sonderdrucke sind zu richten an: Verlag Chemie GmbH, Pappelallee 3, Postfach 1260/1280, D-6940 Weinheim, Telefon (06201) 602-0.

\footnotetext{
Die Chemischen Berichte erscheinen monatlich. Der Jahresbezugspreis beträgt 770. - DM zuzügl. Versandgebühr:n. Einzelheft 85. - DM. In diesem Preis ist die Mehrwertsteuer enthalten. Die Bezugsbedingungen für Mitglieder der Gesellschaft Deutscher Chemiker werden auf Anfrage von der Geschăftsstelle, Carl-Bosch-Haus, Varrentrappsirißc 40-42, Postfach 900440, D-6000 Frankfurt 90, mitgeteilt. - Bestellungen richten Sie bitte an Ihre Fachbuchhandltng oder unmittelbar an den Verlag. - Abbestellungen nur bis spätestens 3 Monate vor Ablauf des Kalenderjahres. - Die Lieferung erfolgt auf Rechnung und Gefahr des Empfängers. Gerichtsstand und Erfüllungsort: Weinheim/Bergstr

Verlag und Anzeigenabteilung: Verlag Chemie GmbH, Postfach 1260/1280, D-6940 Weinheim. Telefon (06201) 60:-0, Telex 465516 vchwh d, Telefax (06201) 602328.

For U.S.A., Canada, Mexico, Central and South America:

Published monthly by Verlag Chemie GmbH, Weinheim, Federal Republic of Germany, Annual subscription prie: $\$ 505.00$ including postage. Second-class postage paid at Jamaica, NY 11431. Printed in the Federal Republic of Gernany. Air-freighted and mailed in the United States by Publications Expediting Inc., 200 Meacham Avenue, Elmoit, NY 11003. Subscribers should place their orders through Verlag Chemie International Inc., 303 N.W. 12th Avente, Deerfield Beach, Florida 33441-1705, U.S.A.
}

U.S. Postmasters: Send address changes for Chemische Berichte to Verlag Chemie International Inc., 303 N.W. I:th Avenue, Deerfield Beach, FL 33441-1705. 


\section{Jahrgang 1984}

\section{ANORGANISCHE CHEMIE}

Herberhold Max, Ehrenreich Wolfgang und Guldner Karlheinz: Schwefeldiimide mit Organoelement-Substituenten der 5 . Hauptgruppe $\ldots \ldots \ldots \ldots \ldots \ldots \ldots \ldots \ldots$

Appel Rolf, Haubrich Gerhard und Knoch Falk: Ambivalentes Verhalten in $\alpha$-Stellung lithiierter Phosphane gegenüber Elektrophilen $\ldots \ldots \ldots \ldots \ldots \ldots \ldots \ldots \ldots$

Maringgele Walter, Sheldrick George M., Meller Anton und Noltemeyer Mathias: Umsetzungen von Metall- und Metalloidverbindungen mit mehrfunktionellen Molekülen, XXXVIII: Reaktion von Ethandiamiden mit Bromdimethylboran .............

Nöth Heinrich und Storch Wolfgang: Beiträge zur Chemie des Bors, 138: $N$-Borylierte Derivate von Phosphorsäureamiden: Beispiele intermolekularer Borotropie .......

Buchler Johann Walter, Dreher Christine und Lay Kiong-Lam: Metallkomplexe mit Tetrapyrrol-Liganden, XXXIV: Reaktionen von Nitridochrom(V)- und Nitridomangan(V)-Porphyrinen mit Triphenylphosphan und Trimethylphosphit

Boenigk Winfried und Hägele Gerhard: Eine reduktive Michaelis-Becker-Reaktion: Darstellung und Eigenschaften des (Pentachlorphenyl)phosphonigsäure-diethylesters ...

\section{ORGANISCHE CHEMIE}

Schweikert Otto, Netscher Thomas, McMullen Gabrielle L., Knothe Lothar und Prinzbach Horst: Cyclisch gekreuzt-konjugierte Bindungssysteme, 42: Höhervinyloge

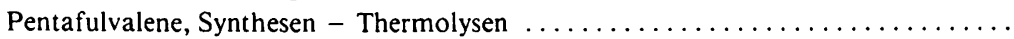

Schweikert Otto, Netscher Thomas, Knothe Lothar und Prinzbach Horst: Cyclisch gekreuzt-konjugierte Bindungssysteme, 43: $(E)$-Sesquifulvatrien, Synthese - Thermo-

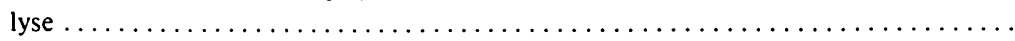

Schweikert Otto, Netscher Thomas, Knothe Lothar und Prinzbach Horst: Cyclisch gekreuzt-konjugierte Bindungssysteme, 44: Höhervinyloge Heptafulvalene, Synthe-

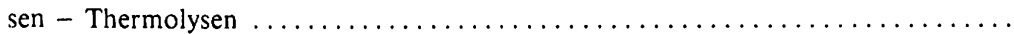

Knupp Gerd und Frahm August W.: Asymmetrische reduktive Aminierung von Cycloalkanonen, 2: Synthese und absolute Konfiguration 2-substituierter Cyclohexanamine

Hünig Siegfried und Prokschy Frank: Azobrücken aus Azinen, V: [3+2]-Cycloadditionen zwischen parallelen $\mathrm{C}=\mathrm{C}$ - und $\mathrm{N}$-alkylierten $\mathrm{N}=\mathrm{N}$-Bindungen $\ldots \ldots \ldots \ldots$

Giese Bernd, Heuck Klaus, Lenhardt Herbert und Lüning Ulrich: Diastereoselektivität

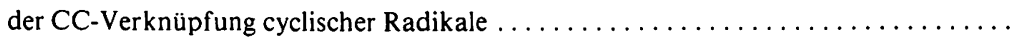

Brokatzky-Geiger Jürgen und Eberbach Wolfgang: Intramolekulare Cycloadditionen mit

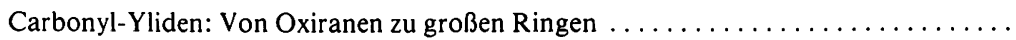

Keul Helmut, Pfeffer Bernd und Griesbaum Karl: Halogenepoxide, 7: Umlagerungsreaktionen bei der Umsetzung von substituierten 2-Chlor- und 2,3-Dichloroxiranen mit Silbertetrafluoroborat ... 
Schaumann Ernst, Wriede Ulrich und Adiwidjaja Gunadi: 1-Cyanalkyliden-substituierte 1,3-Dithietanone als Cyanthioketen-Äquivalente. Umsetzung zu 1,3,5-Dithiazin-

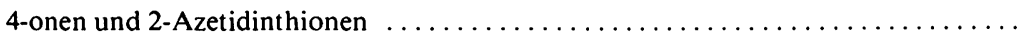

Volkmann Bernhard und Schröder Gerhard: Isolierbare Positionsisomere des Tetrabrombullvalens

Regitz Manfred und Khbeis Saad G.: Untersuchungen an Diazoverbindungen und Aziden, XLIV: 4-Diazomethyl-4H-pyrane durch elektrophile Diazoalkansubstitution

Stahl Ingfried, Schomburg Sabine und Kalinowski Hans Otto: Diastereomere Cyclopropane aus Benzylidensulfuranen und Chalkonen .......................

Fritzsche Katharina und Langhals Heinz: Elektronenreiche Heterocyclen als Donorgruppen in Fluoreszenzfarbstoffen 
INORGANIC CHEMISTRY

Herberhold Max, Ehrenreich Wolfgang, and Guldner Karlheinz: Sulfur Diimides Containing Organoelement Substituents of Main Group $5 \ldots \ldots \ldots \ldots \ldots \ldots \ldots$

Appel Rolf, Haubrich Gerhard, and Knoch Falk: Ambivalent Reactions of Phosphanes

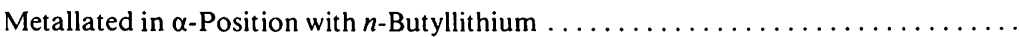

Maringgele Walter, Sheldrick George M., Meller Anton, and Noltemeyer Mathias: Reactions of Metal and Metalloid Compounds with Polyfunctional Molecules, XXXVIII: The Reaction of Ethanediamides with Bromodimethylborane ...............

Nöth Heinrich and Storch Wolfgang: Contributions to the Chemistry of Boron, 138: $N$-Borylated Derivatives of Phosphoric Amides: Examples of Intermolecular Boro-

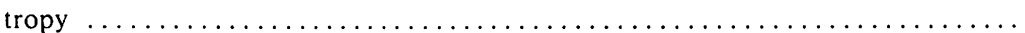

Buchler Johann Walter, Dreher Christine, and Lay Kiong-Lam: Metal Complexes with Tetrapyrrole Ligands, XXXIV: Reactions of Nitridochromium(V)- and Nitridomanganese(V)-Porphyrins with Triphenylphosphane and Trimethylphosphite ........

Boenigk Winfried and Hägele Gerhard: A Reductive Michaelis-Becker Reaction: Synthesis and Properties of Diethyl (Pentachlorophenyl)phosphonite ................

\section{ORGANIC CHEMISTRY}

Schweikert Otto, Netscher Thomas, McMullen Gabrielle L., Knothe Lothar, and Prinzbach Horst: Cyclic Cross-Conjugated Bond Systems, 42: Higher Vinylogous Pentafulvalenes, Syntheses - Thermolyses $\ldots \ldots \ldots \ldots \ldots \ldots \ldots \ldots \ldots \ldots \ldots \ldots \ldots$

Schweikert Otto, Netscher Thomas, Knothe Lothar, and Prinzbach Horst: Cyclic CrossConjugated Bond Systems, 43: $(E)$-Sesquifulvatriene, Synthesis - Thermolysis ....

Schweikert Otto, Netscher Thomas, Knothe Lothar, and Prinzbach Horst: Cyclic CrossConjugated Bond Systems, 44: Higher Vinylogous Heptafulvalenes, Syntheses -

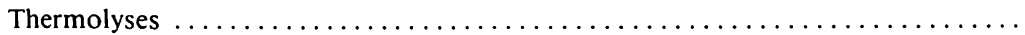

Knupp Gerd and Frahm August W.: Asymmetric Reductive Amination of Cycloalkanones, 2: Synthesis and Absolute Configuration of 2-Substituted Cyclohexanamines

Hünig Siegfried and Prokschy Frank: Azo Bridges from Azines, V: [3+2] Cycloadditions Between Parallel $\mathrm{C}=\mathrm{C}$ and $\mathrm{N}$-Alkylated $\mathrm{N}=\mathrm{N}$ Bonds $\ldots \ldots \ldots \ldots \ldots$

Giese Bernd, Heuck Klaus, Lenhardt Herbert, and Lüning Ulrich: Diastereoselectivity of CC Bond Formation Reaction of Cyclic Radicals

Brokatzky-Geiger Jürgen and Eberbach Wolfgang: Intramolecular Cycloadditions with Carbonyl Ylides: From Oxiranes to Large Ring Systems

Keul Helmut, Pfeffer Bernd, and Griesbaum Karl: Halogen Epoxides, 7: Rearrangements during the Reaction of Substituted 2-Chloro- and 2,3-Dichlorooxiranes with Silver

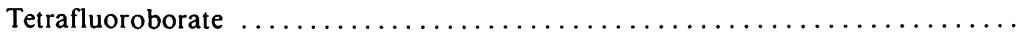

Schaumann Ernst, Wriede Ulrich, and Adiwidjaja Gunadi: 1-Cyanoalkylidene-substituted 1,3-Dithietanones as Cyanothioketene Equivalents. Conversion into 1,3,5-Dithiazin-4-ones and 2-Azetidinethiones $\ldots \ldots \ldots \ldots \ldots \ldots \ldots \ldots \ldots \ldots \ldots \ldots \ldots$ 
Volkmann Bernhard and Schröder Gerhard: Isolatable Positional Isomers of Tetrabromobullvalene

Regitz Manfred and Khbeis Saad G.: Investigations on Diazo Compounds and Azides, XLIV: 4-Diazomethyl-4 $H$-pyrans by Electrophilic Diazoalkane Substitution . . . . .

Stahl Ingfried, Schomburg Sabine, and Kalinowski Hans Otto: Generation of Diastereomeric Cyclopropanes from Benzylidenesulfuranes and Chalcones

Fritzsche Katharina and Langhals Heinz: Electron Rich Heterocycles as Donor Groups in Fluorescent Dyes 


\section{AUTORENREGISTER}

Adiwidjaja, G. s. Schaumann, E. .... 2205

Appel, R., Haubrich, G. und Knoch, F. 2063

Boenigk, W. und Hägele, G. . . . . . . 2287

Brokatzky-Geiger, J. und Eberbach, W. 2157

Buchler, J. W., Dreher, $C$. und

Lay, K.-L. . . . . . . . . . . . . . 2261

Dreher, C. s. Buchler, J. W. . . . . . 2261

Eberbach, W. s. Brokatzky-Geiger, J. . 2157

Ehrenreich, M. s. Herberhold, M. .... 1999

Frahm, A. W. s. Knupp, G. ........ 2076

Fritzsche, K. und Langhals, H. . . . . 2275

Giese, B., Heuck, K., Lenhardt, $H$. und Lüning, $U . \ldots \ldots \ldots \ldots \ldots \ldots 2132$

Griesbaum, K. s. Keul, H. ......... 2193

Guldner, K. s. Herberhold, M. . . . . . . 1999

Hägele, G. s. Boenigk, W. . . . . . . 2287

Haubrich, G. s. Appel, R. . ........ 2063

Herberhold, M., Ehrenreich, W. und Guldner, K. . . . . . . . . . . . . . . 1999

Heuck, K. s. Giese, B. 2132

Hünig, S. und Prokschy, F. . . . . . . . . 2099

Kalinowski, H. O. s. Stahl, I. . . . . . 2247

Keul, H., Pfeffer, $B$. und

Griesbaum, $K$. 2193

Khbeis, S. G. s. Regitz, $M$. 2233

Knoch, F. s. Appel, R. 2063

Knothe, L. s. Schweikert, O. 2006,2027, 2045

Knupp, G. und Frahm, A. W. . . . . . 2076

Langhals, $H$. s. Fritsche, $K$.

2275

Lay, K.-L. s. Buchler, J. W.
Lenhardt, H. s. Giese, B. 2132

Lüning, $U$. s. Giese, $B$. 2132

Maringgele, W., Sheldrick, G. M.,

Meller, A. und Noltemeyer, $M . \quad$. . 2112 McMullen, G. L. s. Schweikert, O. . .. 2006 Meller, A. s. Maringgele, W. ....... 2112 Netscher, T. s.

Schweikert, $O$. 2006, 2027, 2045

Nöth, H. und Storch, W. . . . . . . 2140

Noltemeyer, M. s. Maringgele, W. . . . 2112

Pfeffer, B. s. Keul, H. .......... 2193

Prinzbach, $H$. s.

Schweikert, O. ..... 2006, 2027, 2045

Prokschy, F. s. Hünig, S. . . . . . . . 2099

Regitz, M. und Khbeis, S. G. ....... 2233

Schaumann, E., Wriede, $U$. und

Adiwidjaja, G. ........... 2205

Schomburg, S. s. Stahl, I. . . . . . . . 2247

Schröder, G. s. Volkmann, B. . . . . . . 2226

Schweikert, O., Netscher, T., Knothe, L. und Prinzbach, $H$. 2027,2045

-, Netscher, T., McMullen, G. L.

Knothe, L. und Prinzbach, H. . . . 2006

Sheldrick, G. M. s. Maringgele, W. . . . 2112

Stahl, I., Schomburg, S. und

Kalinowski, H. O. . ........ 2247

Storch, W. s. Nöth, H. . . . . . . . 2140

Volkmann, B. und Schröder, G. . . . . 2226

Wriede, U. s. Schaumann, E. ....... 2205 
Chem. Ber. 117, 2275-2286 (1984)

\title{
Elektronenreiche Heterocyclen als Donorgruppen in Fluoreszenzfarbstoffen
}

\author{
Katharina Fritzsche ${ }^{1)}$ und Heinz Langhals* \\ Institut für Organische Chemie und Biochemie der Universität Freiburg, \\ Albertstr. 21, D-7800 Freiburg i. Br.
}

Eingegangen am 1. September 1983

Die Darstellung von N-Methylphthalimiden wird beschrieben, die in 4-Stellung die Donorgruppen Pyrazol, Triazol, Benzotriazol und Naphthotriazol tragen $(17,18,14,12)$. Über die alkalische Hydrolyse des Imids werden die Substituentenkonstanten der Reste bestimmt. Die Solvatochromie in Absorption und Fluoreszenz gibt Information über die Ladungsverteilung in Grund- und angeregtem Zustand.

\section{Electron Rich Heterocycles as Donor Groups in Fluorescent Dyes}

The synthesis of $N$-methylphthalimides substituted with the donor groups pyrazole, triazole, benzotriazole, and naphthotriazole in position 4 is described $(17,18,14,12)$. The substituent constants of these groups are obtained by alkaline hydrolysis of the corresponding phthalimide dyes. Their solvatochromism in absorption and fluorescence gives information about charge distribution in ground- and excited state.

Eine wichtige Klasse von Farbstoffen ist nach dem Donor-Acceptor-Prinzip 1 oder 2 aufgebaut ${ }^{2-5)}$, nach dem eine elektronenreiche Donorgruppe D über ein konjugiertes System X mit einem elektronenarmen Acceptor A verbunden ist.

$$
\begin{array}{cc}
D-X-A & D-X-A-X-D \\
1 & 2
\end{array}
$$

Während für den Acceptor $\mathrm{A}$ in Form des (partiellen) Carbenium- oder auch Nitrenium-Ions eine universelle Lösung gefunden worden ist, stellt die Entwicklung optimaler Donor-Gruppen noch ein ungelöstes Problem dar. So hat eine Amino-Gruppe zwar eine starke Donor-Wirkung, doch neigt sie z. B. zu Elektronenübertragungsreaktionen, wovon die Photostabilität der Farbstoffe ungünstig beeinflußt wird. Störend ist auch die große Basizität dieser Gruppe, die sich in einer unerwünschten $\mathrm{pH}$-Empfindlichkeit des Farbstoffs äußert.

Die Einführung von elektronenreichen Heterocyclen als Donor-Gruppen brachte hier einen wesentlichen Fortschritt ${ }^{5)}$, denn durch das Einbinden der Donor-Funktion in ein heterocyclisches aromatisches System konnten einige Eigenschaften der Farbstoffe erheblich verbessert werden. Dieses Prinzip wird üblicherweise heute bei optischen Aufhellern angewendet ${ }^{5}$. Die heterocyclischen Gruppen führen in vielen Fällen außerdem zu einer Steigerung der Brillanz und der Fluoreszenzquantenausbeute. 
Erstaunlicherweise ist aber nur wenig Genaues über die Wirkung dieser heterocyclischen Substituenten bekannt, von denen eine Reihe in Schema 1 aufgeführt ist (vgl. Lit. $\left.{ }^{6)}\right)$. Um ihre Donor-Wirkung quantitativ zu erfassen, wäre es insbesondere von Interesse, ihre Substituentenkonstanten zu kennen. Weitere Information kann die Solvatochromie von Farbstoffsystemen liefern, die diese Substituenten tragen ${ }^{7}$.

Schema 1. Heterocyclische Donor-Gruppen in Farbstoffen ${ }^{5)} . X=$ Farbstoff-Rest<smiles>[X]n1nc2ccc3ccccc3c2n1</smiles>

3<smiles>[X]n1nc2cc(C)c(OCCCCCC)cc2n1</smiles>

4<smiles>[X]n1nc(C)c(SCCC)n1</smiles>

$\mathbf{5}$<smiles>[X]n1ccc(C)n1</smiles>

6

Ein geeignetes einfaches Farbstoff-Grundsystem zur Untersuchung der Wirkung von Donor-Substituenten ist das in 4-Stellung substituierte $N$-Methylphthalimid (7), dessen Amino-Derivat (8) im Grundzustand bekanntlich ${ }^{8)}$ nur wenig polar ist, im ersten angeregten Zustand aber eine ausgeprägte Ladungstrennung aufweist ${ }^{8)} .8$ ist in der Absorption $^{7,9)}$ und in der Fluoreszenz ${ }^{7,9,10)}$ solvatochrom und fluoresziert bei erheblicher Photostabilität mit hohen Quantenausbeuten. Darüber hinaus ermöglicht die alkalische Hydrolyse $^{(1)}$ des Farbstoffs, die Substituentenkonstanten der Donorgruppen in 4-Stellung zu bestimmen.<smiles>[2H]c1ccc2c(c1)C(=O)N(C)C2=O</smiles>

7<smiles>CN1C(=O)c2ccc(N)cc2C1=O</smiles>

8

\section{Ergebnisse und Diskussion}

Modellfarbstoff ist das in 4-Stellung substituierte N-Methylphthalimid (7), DonorSubstituenten sind die heterocyclischen Reste von Schema 1. Ausgangsmaterial für alle dargestellten Modell-Farbstoffe ist 4-Amino- $N$-methylphthalimid (8), das aus Phthalimid leicht zugänglich ist. Dessen Nitrierung ${ }^{12,13)}$ führt im Gegensatz zu Phthalsäureanhydrid $^{14)}$ zum reinen 4-Nitro-Derivat 9a, das über eine $S_{N} 2$-Reaktion mit Methyliodid in hohen Ausbeuten zum $N$-Methyl-Derivat 9 b umgesetzt wird ${ }^{15)}$. Dessen Reduktion nach Stephen liefert schließlich das Zwischenprodukt $8^{11,12)}$.<smiles>O=C1NC(=O)c2ccccc21</smiles>

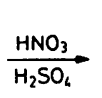<smiles>O=C1NC(=O)c2cc([N+](=O)[O-])ccc21</smiles>

9 a

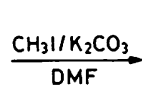<smiles>CN1C(=O)c2ccc([N+](=O)[O-])cc2C1=O</smiles>

\%b

Zur Darstellung des Naphthotriazol-Derivats 12 (vgl. 3) wird 8 diazotiert und entsprechend Schema 2 mit 2-Amino-1-naphthalinsulfonsäure (Tobiassäure) gekuppelt. Durch die ipso-Substitution des Sulfonsäurerestes wird eine Umsetzung mit dem stark 
cancerogenen 2-Naphthylamin vermieden ${ }^{16)}$. Die zuweilen spontane ${ }^{17,18)}$ Oxidation der $o$-Amino-azoverbindungen zu den Triazolen muß im Fall von $\mathbf{1 0}$ mit einem energischen Oxidationsmittel ausgeführt werden. Der übliche Einsatz von alkalischer Hypochloritlösung ${ }^{19 a, b)}$ ist bei $N$-alkylierten Phthalimiden nicht möglich, weil unter den Reaktionsbedingungen spontan Hydrolyse des Phthalimids erfolgt. Unter neutralen Reaktionsbedingungen, unter denen der Phthalimidring hydrolysestabil ist, wird dagegen mit Hypochlorit keinerlei Reaktion mehr festgestellt.

Schema 2. Darstellung der N-Methylphthalimid-Derivate

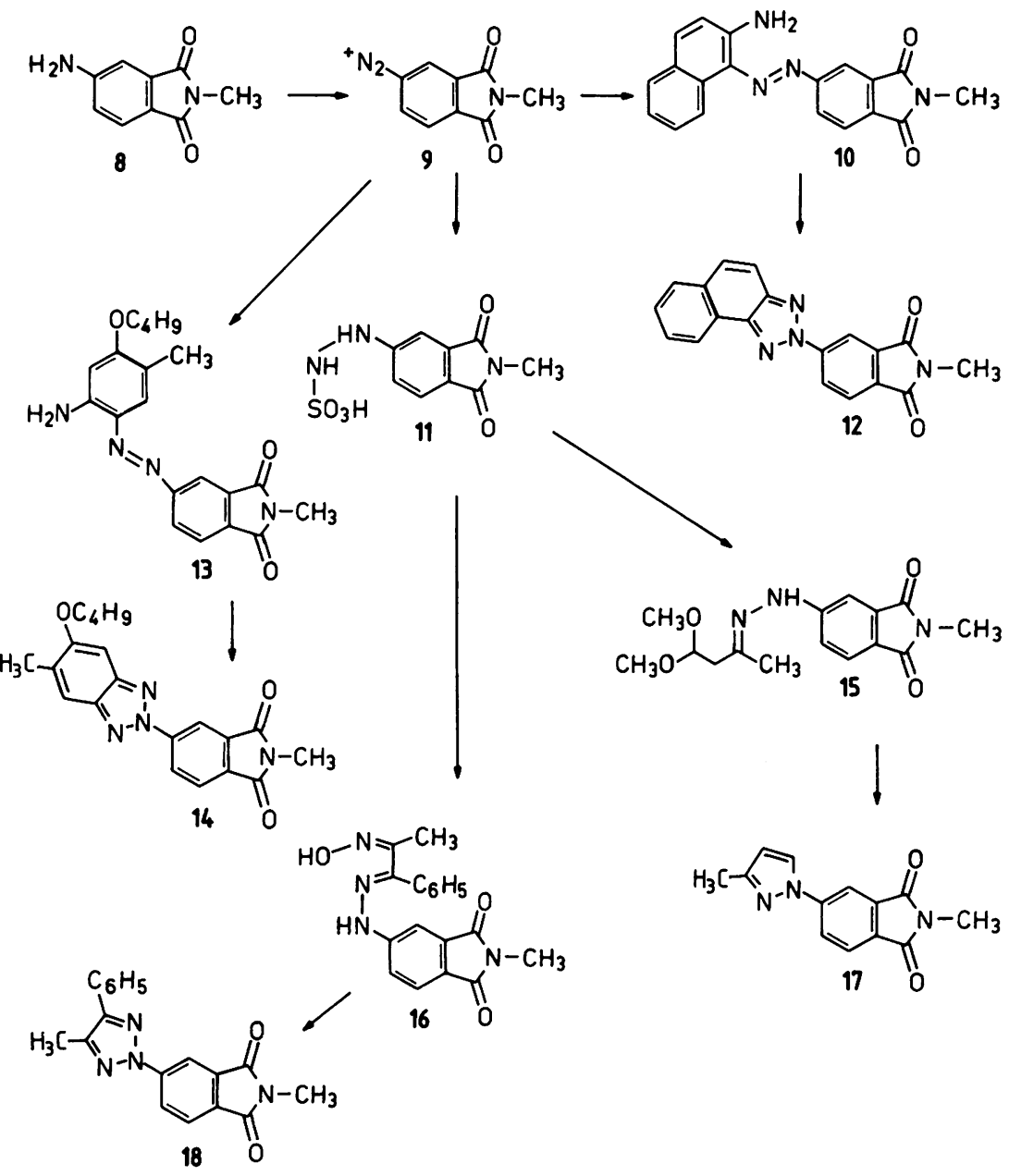

Wir nehmen an, daß im ersten Schritt der Reaktion aus dem Amin ein N-Chloramin entsteht und dieses in einer $\mathrm{S}_{\mathrm{N}}$ 2-analogen Reaktion mit der Azo-Gruppierung cyclisiert . Mit diesem Konzept lassen sich die Reaktionsbedingungen wie folgt verbessern:

Chem. Ber. 117(1984) 
Da sich $N$-Bromamine aus Brom und Aminen sehr leicht bilden und das Bromid eine gute Abgangsgruppe darstellt, wird das Hypochlorit durch $\mathrm{KBr}_{3}$ in gepufferter Lösung ersetzt. Eine weitere Verbesserung bringt der Einsatz des dipolar aprotischen Lösungsmittels Acetonitril $\left(\mathrm{S}_{\mathrm{N}} 2\right)$ als Reaktionsmedium.

Unter diesen modifizierten Reaktionsbedingungen läßt sich nun das Naphthotriazolderivat 12 als kristalline Reinsubstanz mit 44\% Ausbeute darstellen ${ }^{20)}$. Die Synthese des Benzotriazolderivats 14 erfolgt analog zu 12 über die entsprechende Azoverbindung. Eine Umsetzung mit $\mathrm{KBr}_{3}$ zum analogen Benzotriazolderivat gelingt jedoch nicht. Der Amino-substituierte Aromat ist hier bereits so elektronenreich, daß eine elektrophile Kern-Bromierung leichter erfolgt als eine Cyclisierung. Der oxidative Ringschluß ist dagegen mit Kaliumiodat in Eisessig möglich.

Zur Darstellung von 17 und 18 wird zunächst aus 8 das Hydrazin (7, D $\left.=\mathrm{H}_{2} \mathrm{NNH}\right)$ durch Reduktion des Diazoniumsalzes 9 dargestellt. Hierbei erhält man als primäres Reaktionsprodukt das Sulfonsäurehydrazid 11, das wesentlich besser lagerfähig ist als das freie Hydrazin. Es kann an dessen Stelle für die nachfolgenden Umsetzungen eingesetzt werden. Zur Darstellung des Triazols 18 wird 11 mit dem entsprechenden $\alpha$-Oximinoketon ${ }^{19 \mathrm{c})}$ zum $\alpha$-Oximinohydrazon 16 kondensiert, aus dem durch Dehydratisierung mit Acetanhydrid das Triazol 18 entsteht. Zur Darstellung des Pyrazols 17 wird 11 mit dem Acetal des $\beta$-Ketobutyraldehyds kondensiert ${ }^{19 d)}$ und schließlich unter Säurekatalyse cyclisiert.

Alle dargestellten Phthalimidderivate fluoreszieren mit hohen Quantenausbeuten. Ihre spektroskopischen Daten sind in Tab. 1 angegeben.

Wie bereits früher ${ }^{7, y)}$ beschrieben, äußert sich die Solvens-Polarität von Lösungsmitteln bei langsamen und schnellen Prozessen unterschiedlich. Während bei langsamen Prozessen der Orientierung der dominierende Einfluß zukommt (im folgenden Orientierungs-Polarität genannt, mit z. B. der $E_{T}(30)-S k a a^{21)}$ als Maß), kommt bei schnellen Prozessen im wesentlichen die Polarisierbarkeit zum Zuge (z.B. die $\chi_{\mathrm{R}}-$ Skala $^{22)}$ als Maß).

Diese Solvens-Eigenschaften lassen sich nun ihrerseits als Sonde zur Untersuchung des Einflusses von Donor-Substituenten auf die Ladungsverteilung in Grund- und angeregtem Zustand einsetzen.

Als Lösungsmittel hoher Orientierungs-Polarität wird hier Ethanol verwendet (vgl. $\operatorname{den} E_{T}(30)$-Wert) und DMSO als Solvens hoher Polarisierbarkeit (siehe $\chi_{R}$ ). Der unpolare Standard ist Toluol.

Wie bereits bekannt ${ }^{7,9)}$ zeigt das UV/VIS-Spektrum des Amins 8 im leicht polarisierbaren Lösungsmittel DMSO eine besonders starke langwellige Verschiebung der Absorption, während sie bei der Fluoreszenz in Ethanol, also bei hoher Orientierungspolarität, auftritt. Dies steht mit einer geringen Ladungstrennung zwischen Donor und Acceptor im Grundzustand und einer starken im ersten angeregten Zustand ${ }^{8)}$ in Einklang. Die Solvatation des angeregten Zustands, die wegen dessen starker Ladungstrennung den größten Beitrag des Solvenseinflusses liefert, kann wegen des Franck-CondonPrinzips im vorliegenden Beispiel bei der Anregung nur durch Polarisierbarkeitseffekte erfolgen. Die Lebensdauer des angeregten Zustands ist aber so groß, daß für die Fluoreszenz eine Solvatation durch Orientierung des Solvens zum dominierenden Einfluß wird. 
Dieser Effekt tritt in noch stärkerem Maße beim Triazol- (18) und Benzotriazolderivat 14 auf. Man kann daher annehmen, daß der Unterschied der Ladungstrennung zwischen Grund- und erstem angeregten Zustand hier noch größer ist als beim Amin 8. Das Pyrazolderivat 17 zeigt demgegenüber ein entgegengesetztes Verhalten: Das leicht polarisierbare Lösungsmittel DMSO ergibt hier die längstwellige Verschiebung der Fluoreszenz. Hieraus ist zu folgern, daß beim Pyrazol die Ladungstrennung im Grundzustand sogar größer ist als im ersten angeregten Zustand.

Tab. 1. Spektroskopische Daten der Phthalimid-Farbstoffe (Abs. = Absorption, Flu. = Fluoreszenz)

\begin{tabular}{|c|c|c|c|c|c|c|c|}
\hline $\begin{array}{l}\text { Farb- } \\
\text { stoff }\end{array}$ & Solvens & & $\begin{array}{l}\lambda_{\max } \\
{[\mathrm{nm}]}\end{array}$ & $E_{T}^{0 \mathrm{a})}$ & $\sigma^{b)}$ & $\begin{array}{l}\mathrm{ST}^{\mathrm{c})} \\
{[\mathrm{nm}]}\end{array}$ & $T Z^{d)}$ \\
\hline \multirow[t]{3}{*}{8} & Toluol & $\begin{array}{l}\text { Abs. } \\
\text { Flu. }\end{array}$ & $\begin{array}{l}353 \\
440\end{array}$ & $\begin{array}{l}76.8 \\
69.0\end{array}$ & $\begin{array}{l}2.3 \\
1.6\end{array}$ & 42 & 49 \\
\hline & DMSO & $\begin{array}{l}\text { Abs. } \\
\text { Flu. }\end{array}$ & $\begin{array}{l}381 \\
493\end{array}$ & $\begin{array}{l}72.1 \\
60.6\end{array}$ & $\begin{array}{l}3.1 \\
2.2\end{array}$ & 75 & 95 \\
\hline & Ethanol & $\begin{array}{l}\text { Abs. } \\
\text { Flu. }\end{array}$ & $\begin{array}{l}378 \\
522\end{array}$ & $\begin{array}{l}75.4 \\
58.3\end{array}$ & $\begin{array}{l}4.8 \\
1.6\end{array}$ & 111 & 270 \\
\hline \multirow[t]{3}{*}{12} & Toluol & $\begin{array}{l}\text { Abs. } \\
\text { Flu. }\end{array}$ & $\begin{array}{l}372^{j)} \\
402^{f}\end{array}$ & $\begin{array}{l}76.6 \\
73.8\end{array}$ & $\begin{array}{l}1.2 \\
1.1\end{array}$ & 14 & 4.5 \\
\hline & DMSO & $\begin{array}{l}\text { Abs. } \\
\text { Flu. }\end{array}$ & $\begin{array}{l}371.5 \\
464\end{array}$ & $\begin{array}{l}73.9 \\
69.9\end{array}$ & $\begin{array}{l}0.2 \\
2.0\end{array}$ & 22 & 7.6 \\
\hline & Ethanol & $\begin{array}{l}\text { Abs. } \\
\text { Flu. }\end{array}$ & $\begin{array}{l}367 g) \\
484^{f}\end{array}$ & $\begin{array}{l}78.8 \\
72.1\end{array}$ & $\begin{array}{l}2.3 \\
2.3\end{array}$ & 34 & 8.8 \\
\hline \multirow[t]{3}{*}{14} & Toluol & $\begin{array}{l}\text { Abs. } \\
\text { Flu. }\end{array}$ & $\begin{array}{l}356.5^{\mathrm{e})} \\
417\end{array}$ & $\begin{array}{l}76.6 \\
72.5\end{array}$ & $\begin{array}{l}1.5 \\
1.6\end{array}$ & 21 & 6.4 \\
\hline & DMSO & $\begin{array}{l}\text { Abs. } \\
\text { Flu. }\end{array}$ & $\begin{array}{l}367 \\
446^{h)}\end{array}$ & $\begin{array}{l}78.0 \\
67.1\end{array}$ & $\begin{array}{l}2.8 \\
2.1\end{array}$ & 59 & 110 \\
\hline & Ethanol & $\begin{array}{l}\text { Abs. } \\
\text { Flu. }\end{array}$ & $\begin{array}{l}330.5 \\
458\end{array}$ & $\begin{array}{l}85.4 \\
64.4\end{array}$ & $\begin{array}{l}4.4 \\
3.8\end{array}$ & 110 & 640 \\
\hline \multirow[t]{3}{*}{17} & Toluol & $\begin{array}{l}\text { Abs. } \\
\text { Flu. }\end{array}$ & $\begin{array}{l}333 \\
400\end{array}$ & $\begin{array}{l}82.0 \\
75.0\end{array}$ & $\begin{array}{l}2.4 \\
1.9\end{array}$ & 32 & 15 \\
\hline & DMSO & $\begin{array}{l}\text { Abs. } \\
\text { Flu. }\end{array}$ & $\begin{array}{l}332 \\
435\end{array}$ & $\begin{array}{l}82.9 \\
68.6\end{array}$ & $\begin{array}{l}3.3 \\
2.7\end{array}$ & 72 & 240 \\
\hline & Ethanol & $\begin{array}{l}\text { Abs. } \\
\text { Flu. }\end{array}$ & $\begin{array}{l}343^{i)} \\
417\end{array}$ & $\begin{array}{l}82.6 \\
71.9\end{array}$ & $\begin{array}{l}3.9 \\
3.1\end{array}$ & 51 & 10 \\
\hline \multirow[t]{3}{*}{18} & Toluol & $\begin{array}{l}\text { Abs. } \\
\text { Flu. }\end{array}$ & $\begin{array}{l}340 \\
400\end{array}$ & $\begin{array}{l}81.1 \\
75.3\end{array}$ & $\begin{array}{l}2.3 \\
1.4\end{array}$ & 27 & 9.6 \\
\hline & DMSO & $\begin{array}{l}\text { Abs. } \\
\text { Flu. }\end{array}$ & $\begin{array}{l}340.5 \\
462\end{array}$ & $\begin{array}{l}80.8 \\
71.6\end{array}$ & $\begin{array}{l}2.7 \\
0.22\end{array}$ & 46 & 340 \\
\hline & Ethanol & $\begin{array}{l}\text { Abs. } \\
\text { Flu. }\end{array}$ & $\begin{array}{l}337 \\
490\end{array}$ & $\begin{array}{l}83.2 \\
61.0\end{array}$ & $\begin{array}{l}4.5 \\
4.3\end{array}$ & 120 & 620 \\
\hline
\end{tabular}

a) Molare Anregungsenergie für den 0-0-Übergang in $\mathrm{kcal} \cdot \mathrm{mol}^{-1}$, berechnet nach Lit. ${ }^{23)}$. -

b) Halbwertsbreite der Bande in $\mathrm{kcal} \cdot \mathrm{mol}^{-1}$, siehe Lit. ${ }^{23)}$. - c) Spektrale Trennung der 0-0-Banden zwischen Absorption und Fluoreszenz. - d) Trennzahl, siehe Lit. 23). - e) Langwellige Schulter. - f) Kurzwellige Schulter. - g) Schulter bei $354 \mathrm{~nm}$. - h) Stark strukturiertes Spektrum. i) Kurzwelliges Maximum. - j) Weiteres Maximum bei $355 \mathrm{~nm}$.

Besonders deutlich wird das Verhalten der Farbstoffe bei dem Vergleich der Trennzahlen $^{23)}$, die angeben, wie weit das Absorptionsspektrum vom Fluoreszenzspektrum 
spektral getrennt vorliegt. Die Trennzahlen werden mit Hilfe einer Tangentenmetho$\mathrm{de}^{23)}$ aus den Flanken der Spektren bestimmt. Beim Amin (8) steigt die Trennzahl beim Übergang von Toluol zu Ethanol stark an. Dies ist hauptsächlich auf die langwellige Verschiebung der Fluoreszenz im letzteren Lösungsmittel zurückzuführen. Ausgeprägter ist dieser Effekt noch beim Triazol- (18) und beim Benzotriazolderivat (14). Das Pyrazolderivat 17 zeigt dagegen die beste spektrale Trennung im leicht polarisierbaren Lösungsmittel DMSO. Die letzteren Ergebnisse sind von besonderem Interesse für die Entwicklung von Farbstoffen für Fluoreszenz-Solarkollektoren ${ }^{24)}$, bei denen Fluoreszenzfarbstoffe mit guter spektraler Trennung in polymere Matrices eingelagert werden müssen. Hierbei kommt Farbstoffen des Pyrazol-Typs besondere Bedeutung zu, weil Polymermaterialien häufig nur eine geringe Orientierungspolarität besitzen, aber eine große Polarisierbarkeit ${ }^{9,25)}$.

Die Spektren des Naphthotriazolderivats 12 schließlich zeigen keine ausgeprägte Solvensabhängigkeit. Man kann daher annehmen, daß sich die Ladungsverteilungen in Grund- und erstem angeregten Zustand nur wenig unterscheiden. Die bei den Phthalimiden gefundenen Zusammenhänge sollen auch an anderen Farbstoff-Grundgerüsten untersucht werden. Hierüber wird in anderem Zusammenhang berichtet werden.

Die Bestimmung der Substituentenkonstanten von D in 7 erfolgte über die Hydrolysereaktion der Phthalimide. Hierbei entsteht in schneller Reaktion das Monomethylamid-Carboxylat der entsprechenden Phthalsäure ${ }^{11)}$. Die vollständige Hydrolyse zum Dicarboxylat verläuft dagegen wesentlich langsamer. Unter den Reaktionsbedingungen ist ein Abbau der heterocyclischen Reste nicht zu befürchten, da sie gegen Alkali weitgehend beständig sind (vgl. die Soda-Echtheiten der optischen Aufheller) ${ }^{5)}$. Zur Bestimmung der Substituentenkonstanten wird deshalb stets der erste Hydrolyseschritt verwendet, von dem bekannt ${ }^{11)}$ ist, daß er auf Substituenteneffekte empfindlich anspricht. Arbeiten der Literatur ${ }^{11}$ haben diese Reaktion polarographisch verfolgt. Hier wird die apparativ einfachere und sehr präzise UV/VIS-Spektroskopie benutzt.

Da fast alle untersuchten Substanzen eine Donor-Acceptor-Struktur aufweisen und der Acceptor Bestandteil des Imidrings ist, äußert sich eine Hydrolyse in einer erheblichen Veränderung des UV/VIS-Spektrums. Um eine möglichst genaue Messung und Auswertung zu gewährleisten, wird die Hydrolyse in Gegenwart eines großen LaugeÜberschusses vorgenommen. Die Kinetik folgt dann mit hoher Präzision der pseudoersten Ordnung.

Zur Bestimmung des $\rho$-Wertes einer Hammett-Beziehung ${ }^{26)}$ für die Hydrolyse werden außer den dargestellten Farbstoffen noch Phthalimide mit den Resten 4-Chlor, $\mathrm{H}$ und 4-Nitro kinetisch untersucht. Die Ergebnisse sind in Tab. 2 angegeben. Die Hydrolysekinetik der zuletzt genannten Reste läßt sich am besten mit einer HammettBeziehung unter Verwendung der $\sigma_{p}$-Werte beschreiben $\left(\rho=2.7 ; \lg k_{\mathrm{o}}=2.46 ; r=\right.$ 0.997; $\left.\sigma_{\rho}=0.1\right)$. Die $\sigma_{m}$-Werte liefern eine schlechtere Korrelation $(r=0.969)$. Die in Lit. ${ }^{11)}$ angegebene Beziehung mit dem arithmetischen Mittel zwischen $\sigma_{p}$ und $\sigma_{m}$ ergibt bei der Kinetik unter den hier angegebenen Reaktionsbedingungen zwar einen gleich guten Korrelationskoeffizienten wie die $\sigma_{p}$-Werte, die statistische Streuung ist aber größer $\left(r=0.997 ; \sigma_{\rho}=0.2\right)$. Wir sind daher der Auffassung, daß unter den hier angegebenen Bedingungen die $\sigma_{p}$-Werte die beste Beschreibung für den Substituenteneinfluß darstellt. 
Tab. 2. Substituentenkonstanten der heterocyclischen Reste von Schema 1 und Hydrolysekinetiken der Phthalimide

\begin{tabular}{lccccc}
\hline Rest ${ }^{\text {a) }}$ & $\lambda^{\text {b) }}$ & $k \cdot 10^{5}\left[\mathrm{~s}^{-1}\right]^{\mathrm{c})}$ & $r^{\mathrm{d})}$ & $N^{\mathrm{e})}$ & $\sigma_{p}{ }^{\mathrm{n}}$ \\
\hline $\mathrm{NH}_{2}$ & 380 & $6.06 \pm 0.04$ & 0.9997 & 18 & $-0.660 \mathrm{~g})$ \\
$\mathrm{H}$ & 220 & $245 \pm 2$ & 0.9993 & 17 & $0.0 \mathrm{~g})$ \\
$\mathrm{Cl}$ & 305 & $2003 \pm 9$ & 0.9998 & 17 & $0.226 \mathrm{~g})$ \\
$\mathrm{NO}_{2}$ & 285 & $41000 \pm 1000$ & 0.9921 & 17 & $0.778 \mathrm{~g})$ \\
$\mathbf{3}(\mathbf{1 2})$ & 368 & $2360 \pm 10$ & 0.9996 & 17 & 0.309 \\
$\mathbf{4}(\mathbf{1 4})$ & 370 & $2320 \pm 20$ & 0.9996 & 17 & 0.306 \\
$\mathbf{5}(\mathbf{1 8})$ & 340 & $1490 \pm 5$ & 0.9999 & 18 & 0.235 \\
$\mathbf{6}(\mathbf{1 7})$ & 334 & $845 \pm 20$ & 0.9977 & 16 & 0.143 \\
$\mathbf{1 0}^{\mathrm{h}}$ & 530 & $415 \pm 7$ & 0.9978 & 17 & 0.029 \\
$\mathbf{1 3}^{\mathrm{h})}$ & 540 & $318 \pm 2$ & 0.9997 & 17 & -0.014 \\
\hline
\end{tabular}

a) Rest $\mathrm{D}$ in 7. - b) Wellenlänge in $\mathrm{nm}$ bei der Hydrolysekinetik. - ${ }^{c)}$ Geschwindigkeitskonstante der Hydrolyse unter pseudo-erster-Ordnungs-Bedingungen (siehe exp. Teil). - d) Korrelationskoeffizient der Hydrolysekinetik. - e) Anzahl der Meßpunkte. - f Substituentenkonstante berechnet aus $\lg k=2.70 \sigma_{p}-2.46$. - g) Aus Lit. ${ }^{26)}$. - h) Azoverbindungen.

Auf der Grundlage der erhaltenen Hammett-Beziehung lassen sich nun die Substituentenkonstanten der heterocyclischen Reste bestimmen. Die in Tab. 2 angegebenen $\sigma_{p}$-Werte zeigen, daß die in den Farbstoffen verwendeten heterocyclischen Reste sogar eher schwach elektronenziehenden Charakter besitzen, vergleichbar etwa mit Chlor.

Wir nehmen an, daß hierfür der induktive Elektronenzug durch die Stickstoffatome in den Resten verantwortlich ist, der ihre $\pi$-Donorfunktion überkompensiert. Besonders deutlich wird dies beim Vergleich der Substituentenkonstante des Triazols 18 mit der des Pyrazols 17 (zwei bzw. drei Stickstoffe). Es wird dadurch auch verständlich, daß bei Vergleich der Werte von Tab. 1 und 2 kein direkter Zusammenhang zwischen Substituentenkonstante und Wellenlänge der Absorption bzw. Fluoreszenz sowie Umfang der Solvatochromie festgestellt wird. Für die letzteren Größen sind wohl im wesentlichen die $\pi$-Donoreigenschaften der Reste verantwortlich.

Um hierüber noch weitere Informationen zu erhalten, wäre es günstig, die $\sigma_{m}$-Substituentenkonstanten der Reste zu bestimmen. Hierüber soll in anderem Zusammenhang berichtet werden.

Den Herren Prof. H. Balli und C. Rüchardt wird für Anregungen gedankt. Die Arbeit ist von der Stiftung Volkswagenwerk und vom Bundesministerium für Forschung und Technologie gefördert worden, denen hiermit gedankt wird.

\section{Experimenteller Teil}

UV/VIS-Spektren: DMR 21 der Firma Zeiss mit Fluoreszenzzusatz ZMF 4. - 'H-NMR-Spektren: WM 250 der Firma Bruker. - ${ }^{13}$ C-NMR-Spektren: WP 80 der Firma Bruker; angegebene Multiplizitäten beziehen sich auf das off-resonance-Spektrum. - IR-Spektren: PE 457 der Firma Perkin-Elmer. - Massenspektren: SM 1 B der Firma Varian; Bestrahlungslampe: Power Stars HQI-T 2000 W/N der Firma Osram.

N-Methyl-4-nitrophthalimid ${ }^{15)}: \mathrm{Zu} 100 \mathrm{~g}$ (0.52 mol) 4-Nitrophthalimid ${ }^{12,13)}$ und $45 \mathrm{~g}(0.325$ mol) wasserfreiem Kaliumcarbonat in $600 \mathrm{ml}$ wasserfreiem Dimethylformamid (DMF) werden $100 \mathrm{~g}(0.71 \mathrm{~mol})$ Methyliodid gegeben. Innerhalb $1 \mathrm{~h}$ wird die Reaktionstemp. auf $130^{\circ} \mathrm{C}$ gestei-

Chem. Ber. 117(1984) 
gert und dann noch $1 \mathrm{~h}$ bei dieser Temp. belassen. Die abgekühlte Lösung wird auf 21 Eiswasser gegossen, der ausgefallene Feststoff dreimal mit je $400 \mathrm{ml}$ Wasser, $200 \mathrm{ml} 2$ proz. Natronlauge und wieder $400 \mathrm{ml}$ Wasser gewaschen. Der Feststoff wird über Phosphorpentoxid getrocknet und anschließend aus Ethanol umkristallisiert. Ausb. $\left.64 \mathrm{~g} \mathrm{(60 \% ,} \mathrm{Lit.}{ }^{15)} 87.5 \%\right)$, Schmp. $177^{\circ} \mathrm{C}$ (Lit. ${ }^{15)} 177-178^{\circ} \mathrm{C}$ ).

4-Amino-N-methylphthalimid (8) ${ }^{11)}$ : Zu einer Lösung von $160 \mathrm{~g}$ (0.71 mol) Zinn(II)-chloridDihydrat in $115 \mathrm{ml}$ Wasser und $1 \mathrm{l} \mathrm{konz.} \mathrm{Salzsäure}{ }^{12)}$ werden $35 \mathrm{~g}(0.17 \mathrm{~mol}) \mathrm{N}$-Methyl-4-nitrophthalimid gegeben. Die Lösung wird 90 min gerührt und zum Auskristallisieren des Hydrochlorids mit Eis gekühlt. Der Niederschlag wird abgesaugt und über Calciumchlorid getrocknet (93\%). Zur Darstellung des freien Amins wird er mit heißem Wasser zersetzt. Die beim Abkühlen ausfallenden Kristalle werden abgesaugt, über Calciumchlorid getrocknet und aus Ethanol umkristallisiert. Ausb. $21 \mathrm{~g}(70 \%)$, Schmp. $237^{\circ} \mathrm{C}$ (Lit. ${ }^{11)} 242^{\circ} \mathrm{C}$ ), lange, leuchtend gelbgrüne, fluoreszierende Nadeln, $R_{\mathrm{F}}($ Kieselgel/Methylenchlorid $)=0.11$. $-{ }^{1} \mathrm{H}-\mathrm{NMR}\left(\mathrm{CDCl}_{3} /\left[\mathrm{D}_{6}\right] \mathrm{DMSO}\right): \delta=$ $3.05\left(\mathrm{~s} ; 3 \mathrm{H}, \mathrm{CH}_{3} \mathrm{~N}\right), 5.7-5.8\left(\mathrm{~s} ; 2 \mathrm{H}, \mathrm{NH}_{2}\right), 6.82-6.79\left(\mathrm{dd}, J_{5,6}=8.5, J_{3,5}=2.8 \mathrm{~Hz} ; 1 \mathrm{H}, 5-\mathrm{H}\right)$, $7.01\left(\mathrm{~d}, J_{3.5}=2.8 \mathrm{~Hz} ; 1 \mathrm{H}, 3-\mathrm{H}\right), 7.45\left(\mathrm{~d}, J_{5.6}=8.5 \mathrm{~Hz} ; 1 \mathrm{H}, 6-\mathrm{H}\right) .-\mathrm{MS}(70 \mathrm{eV}): m / e=176$ $\left(100 \%, \mathrm{M}^{\oplus}\right), 119(39), 91(48), 64(21), 63$ (29).

2-Methyl-5-nitrobenzolamin (2-Amino-4-nitrotoluol) ${ }^{27)}$ : In $2.11 \mathrm{konz}$. Schwefelsäure werden $210 \mathrm{~g}(1.96 \mathrm{~mol}) o$-Toluidin getropft. Die Mischung wird gerührt, bis sich der anfänglich gebildete Niederschlag auflöst, auf $2^{\circ} \mathrm{C}$ abgekühlt und langsam mit $145 \mathrm{ml}(2.10 \mathrm{~mol}) \mathrm{konz}$. Salpetersäure versetzt, so daß die Temperatur $5^{\circ} \mathrm{C}$ nicht übersteigt. Danach wird auf 4 I Eiswasser gegossen und nach Beendigung der Kristallisation abgesaugt. Die Kristalle werden in 51 warmem Wasser gelöst, die Lösung filtriert und mit Natriumcarbonat neutralisiert. Der dabei ausfallende Feststoff wird abgesaugt, mit Wasser gewaschen, über Calciumchlorid getrocknet und aus Ethanol umkristalli-

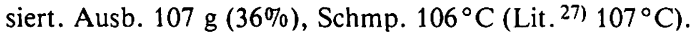

2-Methyl-5-nitrophenol (2-Hydroxy-4-nitrotoluol) ${ }^{27)}: 40 \mathrm{~g}(0.26 \mathrm{~mol})$ 2-Amino-4-nitrotoluol werden in $600 \mathrm{ml} \mathrm{10proz}$. Schwefelsäure unter Erwärmen gelöst. Die klare Lösung wird auf $2{ }^{\circ} \mathrm{C}$ abgekühlt, wobei das Hydrogensulfat des Amins teilweise ausfällt, und langsam mit einer Lösung von $18 \mathrm{~g}(0.26 \mathrm{~mol})$ Natriumnitrit in $50 \mathrm{ml}$ Wasser unter Kühlen versetzt, so daß die Temperatur $5^{\circ} \mathrm{C}$ nicht übersteigt. Die Lösung wird so schnell zu einem siedenden Gemisch von $800 \mathrm{ml}$ Wasser und $400 \mathrm{ml}$ konz. Schwefelsäure getropft, wie es die Stickst offentwicklung erlaubt. Nach beendeter Zugabe wird noch $15 \mathrm{~min}$ in der Hitze gerührt und dann langsam abgekühlt. Die dabei ausgefallenen Kristalle werden abfiltriert und aus Ethanol/Wasser $(40: 60)$ umkristallisiert. Ausb. $26.3 \mathrm{~g}\left(66 \%\right.$, Lit. ${ }^{27)} 85 \%$ ), Schmp. $116^{\circ} \mathrm{C}$ (Lit. ${ }^{27)} 118^{\circ} \mathrm{C}$ ).

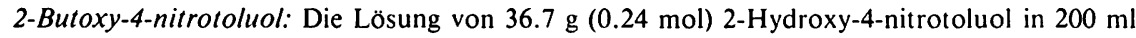
absol. DMF wird mit $33 \mathrm{~g}(0.24 \mathrm{~mol})$ wasserfreiem Kaliumcarbonat versetzt. Unter Stickstoff

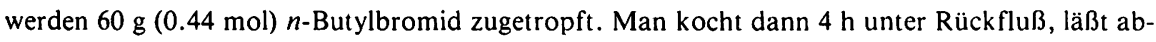
kühlen und gießt auf 41 Eiswasser. Der Niederschlag wird abgesaugt, mit verd. Natronlauge und mit Wasser gewaschen, über Calciumchlorid getrocknet und aus Methanol umkristallisiert. Ausb. $40.6 \mathrm{~g}(81 \%)$, Schmp. $50^{\circ} \mathrm{C}$ (Lit. ${ }^{28)} 51-52^{\circ} \mathrm{C}$ ).

3-Butoxy-4-methylbenzolamin (4-Amino-2-butoxytoluol): Die Lösung von $40.6 \mathrm{~g}(0.19 \mathrm{~mol})$ 2-Butoxy-4-nitrotoluol in $500 \mathrm{ml}$ Ethanol wird innerhalb von $1 \mathrm{~h}$ bei $60^{\circ} \mathrm{C}$ in eine Lösung aus $200 \mathrm{~g}(0.89 \mathrm{~mol})$ Zinn(II)-chlorid-Dihydrat, $200 \mathrm{ml}$ Wasser und $900 \mathrm{ml}$ konz. Salzsäure getropft. Es wird noch $30 \mathrm{~min}$ in der Wärme gerührt und das beim Abkühlen auskristallisierende Hydrochlorid abgesaugt, aus Wasser umkristallisiert und über Calciumchlorid getrocknet. Ausb. $28 \mathrm{~g}$ (68\%), Schmp. $205^{\circ} \mathrm{C}$. Zur Darstellung des freien Amins werden $5.0 \mathrm{~g}$ des Hydrochlorids in $50 \mathrm{ml}$ Wasser in der Wärme gelöst und mit konz. Natriumcarbonatlösung neutralisiert. Das abgeschiedene Amin wird mit Methylenchlorid extrahiert und i. Vak. destilliert. Sdp. $127^{\circ} \mathrm{C} / 0.4$ Torr 
(Lit. ${ }^{29)} 127 / 0.4$ Torr). - ${ }^{1} \mathrm{H}-\mathrm{NMR}\left(\mathrm{CDCl}_{3}\right): \delta=0.93\left(\mathrm{t}, J=6.2 \mathrm{~Hz} ; 3 \mathrm{H}, \mathrm{CH}_{3} \mathrm{C}_{3} \mathrm{H}_{6} \mathrm{O}\right)$, $1.24-1.86\left(\mathrm{~m} ; 4 \mathrm{H}, \mathrm{CH}_{3} \mathrm{C}_{2} \mathrm{H}_{4}\right), 3.71\left(\mathrm{t}, J=5.2 \mathrm{~Hz}, 2 \mathrm{H}, \mathrm{C}_{3} \mathrm{H}_{7} \mathrm{CH}_{2} \mathrm{O}\right), 2.03\left(\mathrm{~s} ; 3 \mathrm{H}, 4-\mathrm{CH}_{3}\right), 3.33$ $\left(\mathrm{s} ; 2 \mathrm{H}, \mathrm{NH}_{2}\right), 5.90\left(\mathrm{~d}, J_{2,6}=1.1 \mathrm{~Hz} ; 1 \mathrm{H}, 2-\mathrm{H}\right), 5.97\left(\mathrm{dd}, J_{5,6}=8.5, J_{2,6}=1.1 \mathrm{~Hz} ; 1 \mathrm{H}, 6-\mathrm{H}\right)$, $6.64\left(\mathrm{~d}, J_{5,6}=8.5 \mathrm{~Hz} ; 1 \mathrm{H}, 5-\mathrm{H}\right)$.

4-(2-Amino-1-naphthyldiazenyl)-N-methylphthalimid (10): Eine Lösung von $6.0 \mathrm{~g} \mathrm{(30} \mathrm{mmol)}$ 4-Amino- $N$-methylphthalimid (8) in $60 \mathrm{ml}$ halbkonz. Salzsäure wird mit $2.0 \mathrm{~g}$ (30 mmol) Natriumnitrit in $10 \mathrm{ml}$ Wasser diazotiert und anschließend langsam unter Eiskühlung in eine Lösung

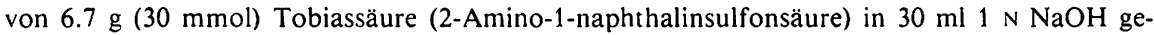
tropft. Der dabei ausfallende Niederschlag wird abgesaugt, über Calciumchlorid getrocknet und mit Ethanol extrahiert. Ausb. $8.3 \mathrm{~g}(84 \%)$, Schmp. $275^{\circ} \mathrm{C}, R_{\mathrm{F}}$ (Kieselgel/Methylenchlorid) $=$ 0.03. - IR (KBr): $3390(\mathrm{NH}), 1760(\mathrm{C}=\mathrm{O}), 1700(\mathrm{C}=\mathrm{O}), 1620(\mathrm{~N}-\mathrm{CO}), 1440,1360,1335$, $1260,1185,740 \mathrm{~cm}^{-1}$. - ${ }^{1} \mathrm{H}-\mathrm{NMR}\left(\mathrm{CDCl}_{3}\right): \delta=3.23\left(\mathrm{~s} ; 3 \mathrm{H}, \mathrm{CH}_{3}\right), 6.91\left(\mathrm{~d}, J_{3^{\prime}, 4^{\prime}}=9.0 \mathrm{~Hz} ; 1 \mathrm{H}\right.$, $\left.3^{\prime}-\mathrm{H}\right), 7.37-7.43\left(\mathrm{~m}, J_{6^{\prime}, 5^{\prime}}=8.2, J_{6^{\prime}, 7^{\prime}}=7.2, J_{6^{\prime}, 8^{\prime}}=1.2 \mathrm{~Hz} ; 1 \mathrm{H}, 6^{\prime}-\mathrm{H}\right), 7.59-7.65\left(\mathrm{~m}, J_{7^{\prime}, 8^{\prime}}=\right.$ $\left.8.2, J_{7^{\prime}, 6^{\prime}}=7.2, J_{7^{\prime}, 5^{\prime}}=1.2 \mathrm{~Hz} ; 1 \mathrm{H}, 7^{\prime}-\mathrm{H}\right), 7.68-7.76\left(\mathrm{~m} ; 2 \mathrm{H}, 5^{\prime}-, 8^{\prime}-\mathrm{H}\right), 7.97\left(\mathrm{dd}, J_{5,6}=7.9\right.$, $\left.J_{3,6}=0.5 \mathrm{~Hz} ; 1 \mathrm{H}, 6-\mathrm{H}\right), 8.18\left(\mathrm{dd}, J_{5,6}=7.9, J_{3,5}=2.4 \mathrm{~Hz} ; 1 \mathrm{H}, 5-\mathrm{H}\right), 8.34\left(\mathrm{dd}, J_{3,5}=2.4, J_{3,6}\right.$ $=0.5 \mathrm{~Hz} ; 1 \mathrm{H}, 3-\mathrm{H}), 8.85\left(\mathrm{dd}, J_{3^{\prime}, 4^{\prime}}=9.0, J_{4^{\prime}, 8^{\prime}}=1.1 \mathrm{~Hz} ; 1 \mathrm{H} ; 4^{\prime}-\mathrm{H}\right) .-\mathrm{MS}(70 \mathrm{eV}): m / e=330$ $\left(65 \%, \mathrm{M}^{\oplus}\right), 142(95), 115(100), 75(24)$.

\section{$\mathrm{C}_{19} \mathrm{H}_{14} \mathrm{~N}_{4} \mathrm{O}_{2}$ (330.3) Ber. C 69.08 H 4.27 N 16.96 Gef. C 69.08 H 4.31 N 16.86}

$\mathrm{N}$-Methyl-4-(2 H-naphthol1,2-d]triazol-2-yl)phthalimid (12) ${ }^{20)}$ : Die Lösung von $0.30 \mathrm{~g}(0.90$ mmol) 10 in $150 \mathrm{ml}$ reinstem Acetonitril wird mit einem Phosphatpuffer bestehend aus $960 \mathrm{mg}$ $(8.0 \mathrm{mmol})$ Natriumdihydrogenphosphat, $1.42 \mathrm{~g}(8.0 \mathrm{mmol})$ Dinatriumhydrogenphosphat-Dihydrat und $20 \mathrm{ml}$ Wasser versetzt. Unter Kühlung mit einer Eis-Kochsalz-Mischung wird eine Lösung von $1.8 \mathrm{ml}(34.9 \mathrm{mmol})$ Brom, $540 \mathrm{mg}(4.5 \mathrm{mmol})$ Kaliumbromid, $30 \mathrm{ml}$ Acetonitril und $5 \mathrm{ml}$ Wasser rasch zugetropft. Es wird noch $30 \mathrm{~min}$ gerührt, wobei sich die Lösung hellorange färbt und das Produkt als farbloser Niederschlag ausfällt. Es wird abgesaugt, über Calciumchlorid getrocknet und aus Toluol umkristallisiert. Ausb. $130 \mathrm{mg}(44 \%)$, Schmp. $281{ }^{\circ} \mathrm{C}, R_{\mathrm{F}}$ (Kieselgel/ Methylenchlorid $)=0.41 .-$ IR $(\mathrm{KBr}): 1770(\mathrm{C}=\mathrm{O}), 1700(\mathrm{C}=\mathrm{O}), 1610(\mathrm{~N}-\mathrm{CO}), 1490,1440$, $1420,1370,1000,950,810,750,740 \mathrm{~cm}^{-1}$. - ${ }^{1} \mathrm{H}-\mathrm{NMR}\left(\mathrm{C}_{6} \mathrm{D}_{6}, 69^{\circ} \mathrm{C}\right): \delta=2.68\left(\mathrm{~s} ; 3 \mathrm{H}, \mathrm{CH}_{3}\right)$, $7.17-7.41\left(\mathrm{~m} ; 4 \mathrm{H}, 6^{\prime}-, 7^{\prime}-, 8^{\prime}-, 9^{\prime}-\mathrm{H}\right), 7.23\left(\mathrm{~d}, J_{4^{\prime}, 5^{\prime}}=1.4 \mathrm{~Hz} ; 1 \mathrm{H}, 5^{\prime}-\mathrm{H}\right), 7.46\left(\mathrm{~d}, J_{4^{\prime}, 5^{\prime}}=1.4 \mathrm{~Hz}\right.$; $\left.1 \mathrm{H}, 4^{\prime}-\mathrm{H}\right), 8.26\left(\mathrm{dd}, J_{5,6}=7.9, J_{3,5}=2.1 \mathrm{~Hz} ; 1 \mathrm{H}, 5-\mathrm{H}\right), 8.52\left(\mathrm{dd}, J_{3,6}=1.1, J_{5,6}=7.9 \mathrm{~Hz} ; 1 \mathrm{H}\right.$, 6- $\mathrm{H}$ ), 8.69 (dd, $\left.J_{3,5}=2.1, J_{3,6}=1.1 \mathrm{~Hz} ; 1 \mathrm{H}, 3-\mathrm{H}\right)$.

\section{$\mathrm{C}_{19} \mathrm{H}_{12} \mathrm{~N}_{4} \mathrm{O}_{2}$ (328.3) Ber. C 69.51 H 3.68 N 17.06 Gef. C 69.32 H 3.69 N 17.22}

4-(2-Amino-4-butoxy-5-methylphenyldiazenyl)- $N$-methylphthalimid (13): Aus $3.0 \mathrm{~g} \mathrm{(17} \mathrm{mmol)}$ 8 wird wie bei 10 beschrieben eine Diazoniumsalzlösung hergestellt und unter Eiskühlung zu einer Lösung von $3.6 \mathrm{~g}(17 \mathrm{mmol})$ 4-Amino-2-butoxytoluol-hydrochlorid in $500 \mathrm{ml}$ Wasser getropft. Der sich abscheidende rote Niederschlag wird abgesaugt, über Calciumchlorid getrocknet und aus

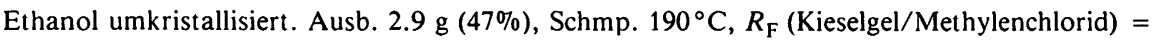
0.23. - IR (KBr): $3400(\mathrm{NH}), 2940,1770(\mathrm{C}=\mathrm{O}), 1700(\mathrm{C}=\mathrm{O}), 1630(\mathrm{~N}-\mathrm{CO}), 1250(\mathrm{C}-\mathrm{O})$, 1210, 1000, $740 \mathrm{~cm}^{-1}$. - UV (Toluol): $\lambda_{\max }(\mathrm{lg} \varepsilon)=477 \mathrm{~nm}(1.75)$. $-{ }^{1} \mathrm{H}-\mathrm{NMR}\left(\mathrm{CDCl}_{3}\right): \delta=$ $1.00\left(\mathrm{t}, J=7.5 \mathrm{~Hz} ; 3 \mathrm{H}, \mathrm{CH}_{3} \mathrm{C}_{3} \mathrm{H}_{6} \mathrm{O}\right), 1.47-1.59\left(\mathrm{~m} ; 2 \mathrm{H}, \mathrm{CH}_{3} \mathrm{CH}_{2}\right), 1.78-1.89(\mathrm{~m} ; 2 \mathrm{H}$, $\left.\mathrm{CH}_{2} \mathrm{CH}_{2} \mathrm{O}\right), 2.16\left(\mathrm{~s} ; 3 \mathrm{H}, \mathrm{CCH}_{3}\right), 3.21\left(\mathrm{~s} ; 3 \mathrm{H}, \mathrm{NCH}_{3}\right), 4.00\left(\mathrm{t}, J=6.6 \mathrm{~Hz} ; 2 \mathrm{H}, \mathrm{CH}_{2} \mathrm{O}\right), 6.11(\mathrm{~s}$; $\left.1 \mathrm{H}, 3^{\prime}-\mathrm{H}\right), 6.29$ (s breit; $\left.2 \mathrm{H}, \mathrm{NH}_{2}\right), 7.59\left(\mathrm{~s} ; 1 \mathrm{H}, 6^{\prime}-\mathrm{H}\right), 7.89\left(\mathrm{~d}, J_{5,6}=7.9 \mathrm{~Hz} ; 1 \mathrm{H}, 6-\mathrm{H}\right), 8.04$ (dd, $\left.J_{3,5}=2.4, J_{5,6}=7.9 \mathrm{~Hz} ; 1 \mathrm{H}, 5-\mathrm{H}\right), 9.18\left(\mathrm{~d}, J_{3,5}=2.4 \mathrm{~Hz} ; 1 \mathrm{H}, 3-\mathrm{H}\right) .-{ }^{13} \mathrm{C}-\mathrm{NMR}\left(\mathrm{CDCl}_{3}\right)$ : $\delta=13.82\left(\mathrm{q} ; \mathrm{CH}_{3} \mathrm{C}_{3} \mathrm{H}_{6} \mathrm{O}\right), 15.29\left(\mathrm{q} ; 5^{\prime}-\mathrm{CH}_{3}\right), 19.35\left(\mathrm{t} ; \mathrm{CH}_{3}-\mathrm{CH}_{2}-\mathrm{C}_{2} \mathrm{H}_{4} \mathrm{O}\right), 24.04\left(\mathrm{q} ; \mathrm{CH}_{3} \mathrm{~N}\right)$, $31.21\left(\mathrm{t} ; \mathrm{CH}_{2} \mathrm{CH}_{2} \mathrm{O}\right.$ ), 68.07 ( $\left.\mathrm{t} ; \mathrm{CH}_{2} \mathrm{O}\right), 97.55$ (d; C-3), 115.33 (d; C-3'), 118.35 (s; C-5), 124.06 (d; C-6'), 127.92, 129.07, 130.51, 131.94, $133.67\left(\mathrm{~s} ; \mathrm{C}-5^{\prime}\right), 144.39$ (s; C-4'), 157.79 (s; C-1), 162.78 (s; $\mathrm{C}-4), 168.26$. $-\mathrm{MS}(70 \mathrm{eV}): m / e=366\left(33 \%, \mathrm{M}^{\oplus}\right), 178(94), 122(100), 57(13)$.

$$
\mathrm{C}_{20} \mathrm{H}_{22} \mathrm{~N}_{4} \mathrm{O}_{3}(366.4) \text { Ber. C } 65.56 \text { H } 6.05 \text { N } 15.29 \text { Gef. C } 65.07 \text { H } 5.76 \text { N } 15.05
$$


4-(5-Butoxy-6-methyl-2 H-benzotriazol-2-yl)-N-methylphthalimid (14): $2.3 \mathrm{~g}(6.3 \mathrm{mmol}) 13$ werden in $500 \mathrm{ml}$ heißer Essigsäure gelöst und schnell in eine siedende Mischung von $5.0 \mathrm{~g}$ ( $23 \mathrm{mmol}$ ) Kaliumiodat in $500 \mathrm{ml}$ Essigsäure getropft. Man läßt 10 min lang abkühlen und gießt dann auf 11 Eis. Der dabei ausfallende Niederschlag (1.5 g) wird abgesaugt, über Calciumchlorid getrocknet und mit Methylenchlorid über eine Kieselgelsäule $(30 \times 3 \mathrm{~cm})$ chromatographiert. Nach $350 \mathrm{ml}$ Eluat mit zwei nicht identifizierten Nebenprodukten wird in $820 \mathrm{ml}$ Methylenchlorid 14 erhalten und aus Toluol umkristallisiert. Ausb. $760 \mathrm{mg}(33 \%)$, Schmp. $186^{\circ} \mathrm{C}, R_{\mathrm{F}}$ (Kieselgel/ Methylenchlorid $)=0.31$. IR $(\mathrm{KBr}): 2930,1770(\mathrm{C}=\mathrm{O}), 1720(\mathrm{C}=\mathrm{O}), 1620(\mathrm{~N}-\mathrm{CO}), 1450$, 1430, 1380, $1280(\mathrm{C}-\mathrm{O}), 1200,1000,960,740 \mathrm{~cm}^{-1}$. ${ }^{1} \mathrm{H}-\mathrm{NMR}\left(\mathrm{CDCl}_{3}\right): \delta=1.02(\mathrm{t}, J=$ $\left.7.5 \mathrm{~Hz} ; 3 \mathrm{H}, \mathrm{CH}_{3} \mathrm{C}_{3} \mathrm{H}_{6} \mathrm{O}\right), 1.51-1.65\left(\mathrm{~m} ; 2 \mathrm{H}, \mathrm{CH}_{3} \mathrm{CH}_{2}\right), 1.83-1.94\left(\mathrm{~m} ; 2 \mathrm{H}, \mathrm{CH}_{2} \mathrm{CH}_{2} \mathrm{O}\right), 2.37$ $\left(\mathrm{s} ; 3 \mathrm{H}, 6^{\prime}-\mathrm{CH}_{3}\right), 3.23\left(\mathrm{~s} ; 3 \mathrm{H}, \mathrm{CH}_{3} \mathrm{~N}\right), 4.07\left(\mathrm{t}, J=6.6 \mathrm{~Hz} ; 2 \mathrm{H}, \mathrm{CH}_{2} \mathrm{O}\right), 6.98\left(\mathrm{~s} ; 1 \mathrm{H}, 4^{\prime}-\mathrm{H}\right), 7.59$ (s; $\left.1 \mathrm{H}, 7^{\prime}-\mathrm{H}\right), 7.97\left(\mathrm{~d}, J_{5,6}=7.9 \mathrm{~Hz} ; 1 \mathrm{H}, 6-\mathrm{H}\right), 8.61\left(\mathrm{dd}, J_{3,5}=2.4, J_{5,6}=7.9 \mathrm{~Hz} ; 1 \mathrm{H}, 5-\mathrm{H}\right), 8.72$ $\left(\mathrm{d}, J_{3,5}=2.4 \mathrm{~Hz} ; 1 \mathrm{H}, 3-\mathrm{H}\right) .-{ }^{13} \mathrm{C}-\mathrm{NMR}\left(\mathrm{CDCl}_{3}\right): \delta=13.90$ und $14.04\left(\mathrm{q} ; \mathrm{CH}_{3} \mathrm{C}_{3} \mathrm{H}_{6} \mathrm{O}\right), 17.99$ und $18.49\left(\mathrm{t} ; \mathrm{CH}_{3} \mathrm{CH}_{2}\right), 19.39$ und $1949\left(\mathrm{q} ; 6^{\prime}-\mathrm{CH}_{3}\right), 24.26\left(\mathrm{q} ; \mathrm{CH}_{3} \mathrm{~N}\right), 31.19$ und 32.38 (t; $\mathrm{CH}_{2} \mathrm{CH}_{2} \mathrm{O}$ ), 68.19 und 73.45 (t; $\mathrm{CH}_{2} \mathrm{O}$ ), 93.92 (d), 114.64 und 115.25 (d), 117.64 und 118.59 (d), 124.37 und 125.10 (d; C-6), 130.51 (s), 131.44 (s), 133.97 und 134.10 (d), 136.32 (d), 141.86 (s), 144.26 (s), 147.85 (s), 158.90 und 159.82 (s), 167.17 (s), 167.43 (s) (Signalverdopplung durch cisoid-transoid-Rotamere um die $\mathrm{C}-\mathrm{N}$-Einfachbindung zwischen den beiden $\pi$-Systemen). MS (70 eV): $m / e=364\left(17 \%, \mathrm{M}^{\oplus}\right), 308(100), 307(18), 174(2), 117(14), 89(15), 62(6), 57$ (13), 38 (3).

\section{$\mathrm{C}_{20} \mathrm{H}_{20} \mathrm{~N}_{4} \mathrm{O}_{3}$ (364.4) Ber. C 65.92 H 5.53 N 15.37 Gef. C 65.90 H 5.43 N 15.42}

2-(2,3-Dihydro-1,3-dioxo-2-methyl-1 H-isochinolin-5-yl)-1-hydrazinsulfonsäure (11): $24 \mathrm{~g} \mathrm{(0.12}$ mol) 4-Amino- $N$-methylphthalimid-hydrochlorid $(8 \cdot \mathrm{HCl})$ werden in $250 \mathrm{ml}$ 16proz. Salzsäure gelöst und wie bei 10 beschrieben mit einer Lösung von $11 \mathrm{~g}(0.16 \mathrm{~mol})$ Natriumnitrit in $20 \mathrm{ml}$ Wasser diazotiert. Durch langsames Zutropfen von ca. $350 \mathrm{ml}$ konz. Natriumcarbonat-Lösung unter Eiskühlung wird der pH-Wert der Diazoniumsalzlösung auf 6- 7 eingestellt. Danach wird die Lösung unter Rühren rasch in eine Lösung von $55 \mathrm{~g}(0.44 \mathrm{~mol})$ Natriumsulfit in $400 \mathrm{ml}$ Wasser getropft. Nach $30 \mathrm{~min}$ wird mit $100 \mathrm{ml}$ konz. Salzsäure angesäuert und $1 \mathrm{~h}$ auf $100^{\circ} \mathrm{C}$ erhitzt. Die abgekühlte Lösung wird i. Vak. auf ca. $500 \mathrm{ml}$ eingeengt, wobei 11 in hellgelben Flocken ausfällt. Es wird abfiltriert, über Calciumchlorid getrocknet und unter Stickstoff (in Lösung hohe Oxidationsempfindlichkeit) aus Ethanol/Wasser umkristallisiert. Das Reaktionsprodukt kann noch

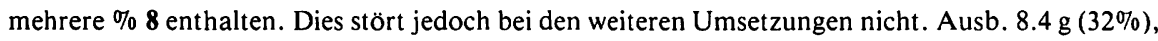
Schmp. $210^{\circ} \mathrm{C}$ (Zers.). - IR (KBr): $3500-2600(\mathrm{OH}, \mathrm{NH}), 1755(\mathrm{C}=\mathrm{O}), 1690(\mathrm{C}=\mathrm{O}), 1610$ $(\mathrm{N}-\mathrm{CO}), 1430,1380,1235,1190,1040,740 \mathrm{~cm}^{-1} .-{ }^{1} \mathrm{H}-\mathrm{NMR}\left(\mathrm{D}_{2} \mathrm{O} / \mathrm{DSS}\right): \delta=3.01(\mathrm{~s} ; 3 \mathrm{H}$, $\left.\mathrm{CH}_{3} \mathrm{~N}\right), 7.17\left(\mathrm{dd}, J_{4,6}=2.1, J_{6,7}=8.1 \mathrm{~Hz} ; 1 \mathrm{H}, 6-\mathrm{H}\right), 7.27\left(\mathrm{~d}, J_{4,6}=2.1 \mathrm{~Hz} ; 1 \mathrm{H}, 4-\mathrm{H}\right), 7.56(\mathrm{~d}$, $\left.J_{6,7}=8.1 \mathrm{~Hz} ; 1 \mathrm{H}, 7-\mathrm{H}\right)$.

\section{$\mathrm{C}_{9} \mathrm{H}_{9} \mathrm{~N}_{3} \mathrm{O}_{5} \mathrm{~S}$ (271.3) Ber. C 39.85 H 3.34 Gef. C 39.55 H 3.37}

N-Methyl-4-(3-methyl-1 H-pyrazol-1-yl)phthalimid (17): Die Lösung von $2.4 \mathrm{~g}(8.9 \mathrm{mmol}) 11$ und $1.1 \mathrm{~g} \mathrm{(8.0} \mathrm{mmol)} \mathrm{3-Oxobutyraldehyd-dimethylacetal} \mathrm{in} 120 \mathrm{ml}$ Ethylenglycol-monoethylether wird unter Stickstoff $1.5 \mathrm{~h}$ unter Rückfluß gekocht. Danach werden $0.2 \mathrm{ml}$ konz. Salzsäure zugegeben, und noch weitere $6 \mathrm{~h}$ wird unter Rückfluß gekocht. Die Lösung wird i. Vak. zur Trockene eingedampft, der Rückstand in Methylenchlorid aufgenommen und die Lösung über eine kurze mit Kieselgel gefüllte Säule filtriert. Das Lösungsmittel wird abgedampft und der Rückstand aus Ethanol umkristallisiert. Ausb. $980 \mathrm{mg}(46 \%)$, Schmp. $201^{\circ} \mathrm{C}, R_{\mathrm{F}}$ (Kieselgel/Methylenchlorid) $=$ 0.09. - IR (KBr): 2920, $1770(\mathrm{C}=\mathrm{O}), 1700(\mathrm{C}=\mathrm{O}), 1610(\mathrm{~N}-\mathrm{CO}), 1540,1490,1430,1370,1240$, $1000,740,580,380 \mathrm{~cm}^{-1}$. - ${ }^{1} \mathrm{H}-\mathrm{NMR}\left(\mathrm{CDCl}_{3}\right): \delta=2.38\left(\mathrm{~s} ; 3 \mathrm{H}, 3^{\prime}-\mathrm{CH}_{3}\right), 3.22\left(\mathrm{~s} ; 3 \mathrm{H}, \mathrm{CH}_{3} \mathrm{~N}\right)$, $6.36\left(\mathrm{~d}, J_{4^{\prime}, 5^{\prime}}=2.7 \mathrm{~Hz} ; 1 \mathrm{H}, 4^{\prime}-\mathrm{H}\right), 7.89\left(\mathrm{dd}, J_{3,6}=0.5, J_{5,6}=7.9 \mathrm{~Hz} ; 1 \mathrm{H}, 6-\mathrm{H}\right), 7.94\left(\mathrm{~d}, J_{4^{\prime}, 5^{\prime}}=\right.$ $\left.2.7 \mathrm{~Hz} ; 1 \mathrm{H}, 5^{\prime}-\mathrm{H}\right), 8.08\left(\mathrm{dd}, J_{3,5}=2.4, J_{5,6}=7.9 \mathrm{~Hz} ; 1 \mathrm{H}, 5-\mathrm{H}\right), 8.1\left(\mathrm{dd}, J_{3,5}=2.4, J_{3,6}=\right.$ 
$0.5 \mathrm{~Hz} ; 1 \mathrm{H}, 3-\mathrm{H}) . \quad-{ }^{13} \mathrm{C}-\mathrm{NMR}\left(\mathrm{CDCl}_{3}\right): \delta=13.75\left(3^{\prime}-\mathrm{CH}_{3}\right), 24.07\left(\mathrm{CH}_{3} \mathrm{~N}\right), 109.39\left(\mathrm{C}-4^{\prime}\right)$, 112.53 (C-3), 122.79 (C-5), 124.64 (C-6), $127.59\left(\mathrm{C}^{\prime} 5^{\prime}\right), 128.46\left(\mathrm{C}-3^{\prime}\right), 134.27$ (C-1), 144.62 (C-2), $152.30(\mathrm{C}-4), 167.70,167.79$. $-\mathrm{MS}(70 \mathrm{eV}): m / e=241\left(100 \%, \mathrm{M}^{\oplus}\right), 197(23), 156(16), 103$ (10), 75 (24).

\section{$\mathrm{C}_{13} \mathrm{H}_{11} \mathrm{~N}_{3} \mathrm{O}_{2}$ (241.2) Ber. C $64.72 \mathrm{H} 4.60$ N 17.42 Gef. C 64.66 H 4.24 N 17.42}

4-\{2-[2-(Hydroxyimino)-I-phenylpropyliden]hydrazino $\}-N$-methylphthalimid (16): $2.3 \mathrm{~g}$ (8.5 mmol) 11 werden mit $1.3 \mathrm{~g}(8.0 \mathrm{mmol}) 1$-Phenyl-1,2-propandion-2-oxim, $0.5 \mathrm{ml}$ Essigsäure, $40 \mathrm{ml}$ Ethanol und $20 \mathrm{ml}$ Wasser $1.5 \mathrm{~h}$ unter Rückfluß gekocht. Nach dem Abkühlen wird das auskristallisierte Produkt abgesaugt und über Calciumchlorid getrocknet. Ausb. $2.3 \mathrm{~g}(55 \%)$, Schmp. $188^{\circ} \mathrm{C}$. - IR (KBr): $3380(\mathrm{OH}), 1755(\mathrm{C}=\mathrm{O}), 1690(\mathrm{C}=\mathrm{O}), 1610(\mathrm{~N}-\mathrm{CO}), 1500,1430,1380$, $1000,740 \mathrm{~cm}^{-1}$.

$\mathrm{C}_{18} \mathrm{H}_{16} \mathrm{~N}_{4} \mathrm{O}_{3} \cdot \mathrm{H}_{2} \mathrm{O}(354.4)$ Ber. C $61.01 \mathrm{H} 5.12 \mathrm{~N} 15.87$ Gef. C $61.42 \mathrm{H} 4.64 \mathrm{~N} 16.14$

N-Methyl-4-(4-methyl-5-phenyl-2H-1,2,3-triazol-2-yl)phthalimid (18): $2.3 \mathrm{~g}(6.5 \mathrm{mmol}) 16,35 \mathrm{ml}$ Acetanhydrid, $1.75 \mathrm{~g}$ wasserfreies Natriumacetat und $1.75 \mathrm{ml}$ Essigsäure werden unter Feuchtigkeitsausschluß 8 h unter Rückfluß gekocht. Die Lösung wird auf Eis gegossen, der Niederschlag abgesaugt, über Calciumchlorid getrocknet, in Methylenchlorid gelöst und die Lösung über eine kurze Kieselgel-Säule filtriert. Das Filtrat wird zur Trockne eingedampft und der Rückstand aus Ethanol umkristallisiert. Ausb. $940 \mathrm{mg}(45 \%)$, Schmp. $177^{\circ} \mathrm{C}, R_{\mathrm{F}}$ (Kieselgel/Methylenchlorid) = 0.34. - IR (KBr): $1770(\mathrm{C}=\mathrm{O}), 1710(\mathrm{C}=\mathrm{O}), 1620(\mathrm{~N}-\mathrm{CO}), 1490,1460,1430,1380,1325,1250$, $1000,950,880,740 \mathrm{~cm}^{-1}$. - ${ }^{1} \mathrm{H}-\mathrm{NMR}\left(\mathrm{CDCl}_{3}\right): \delta=2.36\left(\mathrm{~s} ; 3 \mathrm{H}, 4^{\prime}-\mathrm{CH}_{3}\right), 3.22\left(\mathrm{~s} ; 3 \mathrm{H}, \mathrm{CH}_{3} \mathrm{~N}\right)$, $7.44-7.56(\mathrm{~m} ; 3 \mathrm{H}, m$ - und $p-\mathrm{H}$ in $\mathrm{Ph}), 7.83\left(\mathrm{dd}, J_{o, m}=7.5, J_{o, p}=1.2 \mathrm{~Hz} ; 2 \mathrm{H}, o-\mathrm{H}\right.$ in $\left.\mathrm{Ph}\right), 7.94$ $\left(\mathrm{d}, J_{5,6}=7.9 \mathrm{~Hz} ; 1 \mathrm{H}, 6-\mathrm{H}\right), 8.43\left(\mathrm{dd}, J_{3,5}=2.4, J_{5,6}=7.9 \mathrm{~Hz} ; 1 \mathrm{H}, 5-\mathrm{H}\right), 8.58\left(\mathrm{~d}, J_{3,5}=\right.$ $2.4 \mathrm{~Hz} ; 1 \mathrm{H}, 3-\mathrm{H}) .-{ }^{13} \mathrm{C}-\mathrm{NMR}\left(\mathrm{CDCl}_{3}\right): \delta=12.20\left(\mathrm{q} ; 4^{\prime}-\mathrm{CH}_{3}\right), 24.07\left(\mathrm{q} ; \mathrm{CH}_{3} \mathrm{~N}\right), 113.06(\mathrm{~d}$; C-3), 122.62 (d; C-5), 124.33 (d; C-6), 127.37 (d; $o-C$ in Ph), 128.68 (d; $m-C$ in Ph), 128.87 (d; $p$-C in Ph), 129.35 (s; C-4'), 129.47 (s; C-5'), 134.04 (s; C-1), 143.80 (s; C-1 in Ph), 144.53 (s; $\mathrm{C}-2), 147.84(\mathrm{~s} ; \mathrm{C}-4), 167.44(\mathrm{~s}), 167.67(\mathrm{~s}) .-\mathrm{MS}(70 \mathrm{eV}): m / e=318\left(100 \%, \mathrm{M}^{\oplus}\right), 174(42), 117$ (61), 103 (23), 89 (77), 77 (14), 62 (38), 51 (11), 38 (14).

$$
\mathrm{C}_{18} \mathrm{H}_{14} \mathrm{~N}_{4} \mathrm{O}_{2} \text { (318.3) Ber. C } 67.92 \mathrm{H} 4.43 \text { N } 17.60 \text { Gef. C } 67.45 \mathrm{H} 4.10 \text { N } 17.66
$$

$N$-Methylphthalimid ${ }^{30)}: 100 \mathrm{ml}$ wasserfreies DMF werden mit $9.0 \mathrm{~g}(65 \mathrm{mmol})$ wasserfreiem Kaliumcarbonat, $14.7 \mathrm{~g}$ (100 mmol) Phthalimid und $15.3 \mathrm{~g}$ (107 mmol) Methyliodid versetzt und dann $1.25 \mathrm{~h}$ unter Rückfluß gekocht. Nach dem Erkalten wird auf $400 \mathrm{ml}$ Eiswasser gegossen. Das ausgefallene Produkt wird abgesaugt, mit Eiswasser gewaschen und aus Wasser umkristallisiert (Löslichkeit ca. $1 \mathrm{~g} / 100 \mathrm{ml}$ Wasser). Ausb. $12.43 \mathrm{~g}(77 \%)$ sehr lange, farblose Nadeln, Schmp. $128-129^{\circ} \mathrm{C}$ (Lit. $\left.{ }^{31)} 129-130^{\circ} \mathrm{C}\right)$. $-{ }^{1} \mathrm{H}-\mathrm{NMR}$ ([D $\left.\left.\mathrm{D}_{6}\right] \mathrm{DMSO}\right): \delta=3.00$ (s; $3 \mathrm{H}, \mathrm{NCH}_{3}$ ), 7.71 (s; 4H, Aromaten-H).

Messung der Hydrolysekinetiken von 12, 14, 17, 18: Von den Imiden werden Lösungen in Methylcellosolve (Ethylenglycol-monomethylether) hergestellt, die im UV-Spektrum bei $\lambda_{\max }$ und $1 \mathrm{~cm}$ Schichtdicke eine Extinktion von ca. 1 aufweisen. Von diesen Lösungen werden jeweils $3 \mathrm{ml}$ in eine 1-cm-Quarzküvette gegeben und mit $0.2 \mathrm{ml} 0.1 \mathrm{~N} \mathrm{NaOH}$ versetzt. Dann wird kurz geschüttelt und sofort die Abnahme der UV-Absorption bei $\lambda_{\max }$ gemessen. Die Extinktions-Zeit-Kurven erfüllen das Zeitgesetz der 1. Ordnung mit hoher Präzision und werden wie üblich ausgewertet. Geschwindigkeitskonstanten s. Tab. 2.

Photobleichkinetiken: Messung und Auswertung erfolgten analog zu Lit. ${ }^{32}$. Die Halbwertszeiten der Farbstoffe 12, 14, 18, 17 und 8 verhalten sich wie $1: 1.7: 2.1: 2.3: 3.4$. 
1) K. Fritzsche, Diplomarbeit, Univ. Freiburg 1983.

2) J. Griffiths, Colour and Constitution of Organic Molecules, 1. Aufl., Academic Press, London 1976.

3) J. Fabian und H. Hartmann, Light Absorption of Organic Colorants, 1. Aufl., Springer, Berlin 1980.

4) E. N. Abrahart, Dyes and their Intermediates, 2. Aufl., Ewald Arnold, London 1977.

5) $R$. Anliker und $G$. Müller in F. Coulston und F. Korte, Fluorescent Whitening Agents, 1. Aufl., Georg Thieme, Stuttgart 1975.

6) $R$. Naef, Habilitationsschrift, Univ. Basel 1982 .

7) $H$. Langhals, Angew. Chem. 94, 739 (1982); Angew. Chem., Int. Ed. Engl. 21, 724 (1982).

8) 8a) V. A. Palm, Grundlagen der quantitativen Theorie organischer Reaktionen, Akademie Verlag, Berlin 1971. - 8b) I. A. Koppel und V. A. Palm in N. B. Chapman und J. Shorter, Advances in Linear Free Energy Relationships, 1. Aufl., S. 203-280, Plenum, London 1973.

9) $H$. Langhals, Z. Phys. Chem. N.F. 127, 45 (1981).

10) 10a) I. A. Zhmyreva, V. V. Zelinskii, V. P. Kolobkov und N. D. Krasnitskaya, Dokl. Akad. Nauk SSSR 129, 1089 (1959) [Chem. Abstr. 55, $26658 \mathrm{f}$ (1961)]. - 10b) V. V. Zelinskii, V. P. Kolobkov und L. G. Pikulik, Opt. Spektrosk. 1, 161 (1956) [Chem. Abstr. 51, 4418 b (1957)]. - 10c) T. V. Veselova, I. I. Reznikova, A. S. Cherkasov und V. I. Shirokov, Opt. Spectrosk. 25, 98 (1968) [Chem. Abstr. 69, 72593 t (1968)].

11) R. Dabard und J. Tirouflet, Bull. Soc. Chim. Fr. 1957, 565.

12) L. F. Levy und H. Stephen, J. Chem. Soc. 1931, 79.

13) E. Huntress und R. L. Shirner, Org. Synth., Coll. Vol. 2, 459 (1943).

14) W. A. Lawrence, J. Am. Chem. Soc. 42, 1872 (1920).

15) J. H. Billman und R. V. Cash, J. Am. Chem. Soc. 75, 2499 (1953).

16) 16a) CIBA-Geigy (Erf. A. Hagenböcker und M. Blumer), US-Pat. 2776961 (8. 1. 1957) [Chem. Abstr. 51, P $4718 \mathrm{~h}$ (1957)]. - 16b) CIBA-Geigy (Erf. A. Hagenböcker und M. Blumer), Swiss. Pat. 298883 (2. 8. 1954) [Chem. Abstr. 49, 15247 h (1955)].

17) L. Gattermann und G. Wichmann, Ber. Dtsch. Chem. Ges. 21, 1633 (1880).

18) O. N. Witt und G. Schmitt, Ber. Dtsch. Chem. Ges. 27, 2370 (1894).

19) 19a) K. H.Schündehütte in Methoden der organischen Chemie (Houben-Weyl-Müller), 4. Aufl., Bd. X/3, Thieme, Stuttgart 1965. - 19b) J. Dobas, J. Pirkl und V. Hanousek, Collect. Czech. Chem. Commun. 23, 280 (1958). - 19c) R. F. Coles und C. F. Hamilton, J. Am. Chem. Soc. 68, 1799 (1946). - 19d) J. R. Geigy A.G. (Erf. H. Häusermann), Franz. Pat. 1320597 (8. März 1963) [Chem. Abstr. 59, P $14144 \mathrm{~g}$ (1963)].

20) V. Sachweh, unveröffentlichte Studienarbeit.

21) 21a) C. Reichardt, Angew. Chem. 91, 119 (1979); Angew. Chem., Int. Ed. Engl. 18, 98 (1979). - 21b) C. Reichardt, Solvent Effects in Organic Chemistry, 1. Aufl., Verlag Chemie, Weinheim 1979.

22) L. G. S. Brooker, G. H. Keyes und D. W. Heseltine, J. Am. Chem. Soc. 73, 5350 (1951).

23) H. Langhals, Ber. Bunsenges. Phys. Chem. 83, 730 (1979).

24) $H$. Langhals, Nachr. Chem. Tech. Lab. 28, 716 (1980).

25) $H$. Langhals, Angew. Chem. 94, 452 (1982); Angew. Chem., Int. Ed. Engl. 21, 423 (1982).

26) 26a) L. P. Hammett, Chem. Rev. 17, 125 (1935). - 26b) H. H. Jaffe, Chem. Rev. 53, 191 (1953).

27) L. W. Clemence und G. W. Raiziss, J. Am. Pharm. Assoc. 23, 536 (1934).

${ }^{28)} D$. S. Bhate, T. B. Panse und $K$. Venkataraman, Proc. Indian Acad. Sci., Sect. A 32, 357 (1950).

29) Y. Tanaka, J. Sci. Research Inst. Tokyo 48, 192 (1954) [Chem. Abstr. 50, 4834 i (1956)].

30) S. Demmig, unveröffentlichte Studienarbeit.

31) C. Graebe und A. Pictet, Ber. Disch. Chem. Ges. 17, 1173 (1884).

32) A. Rademacher, S. Märkle und H. Langhals, Chem. Ber. 115, 2927 (1982). 